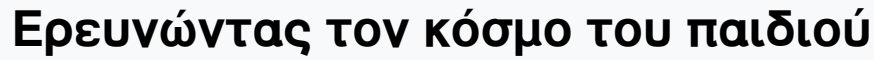

Tóp. 5 (2003)

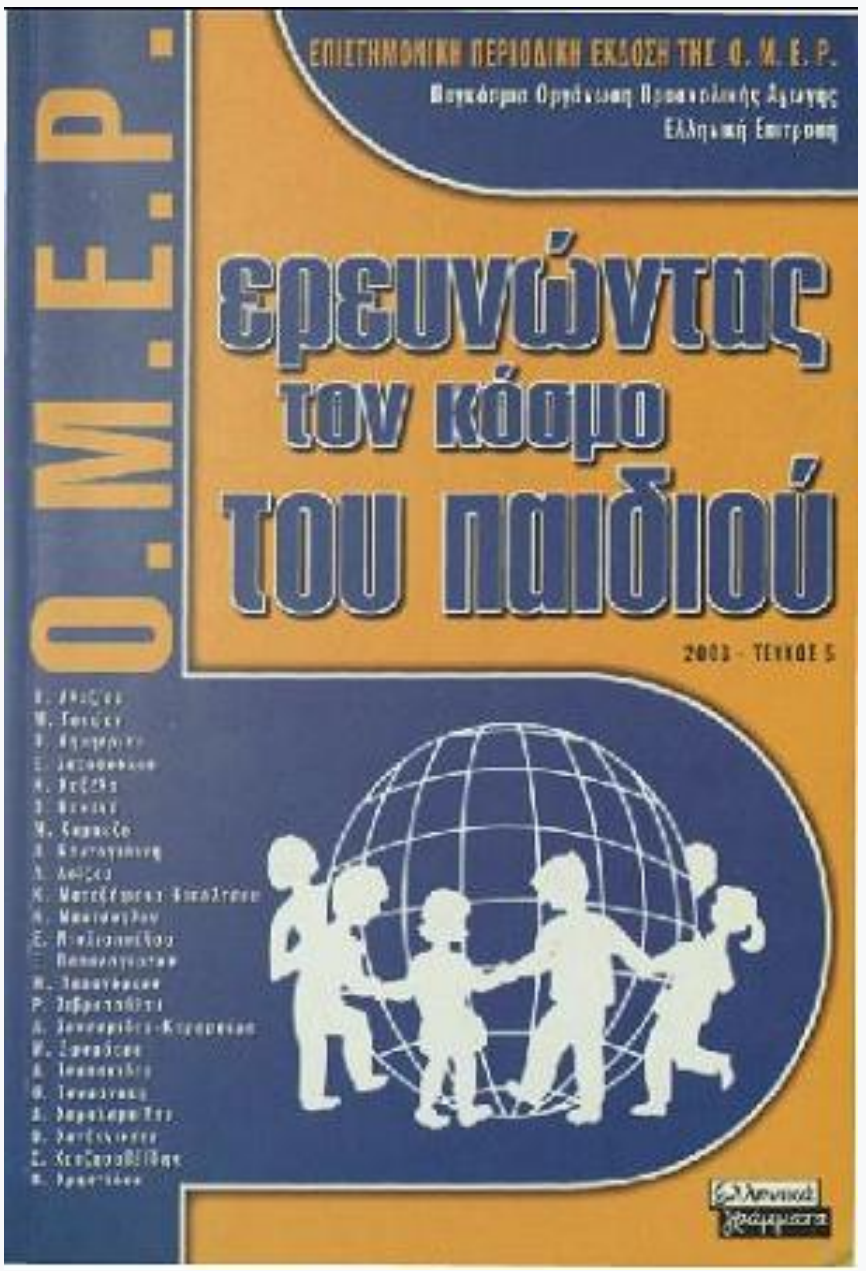

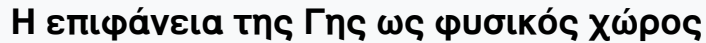

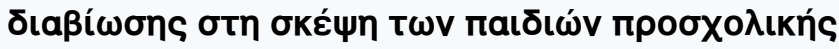

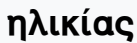

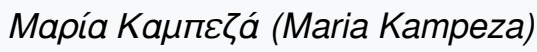

doi: $10.12681 /$ icw.18092

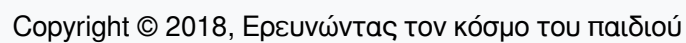

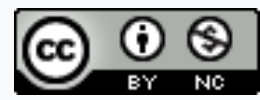

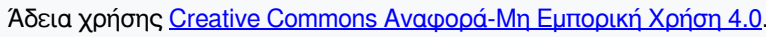

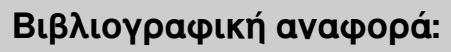

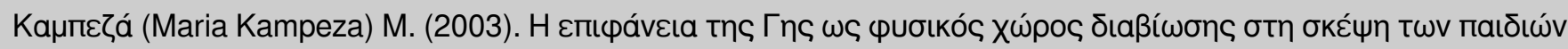

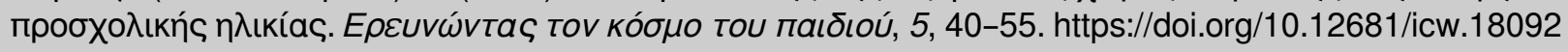




\section{Mapía Карпєろá}

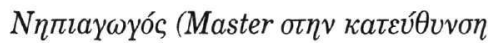

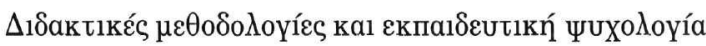

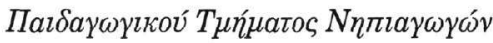

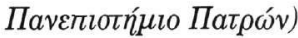

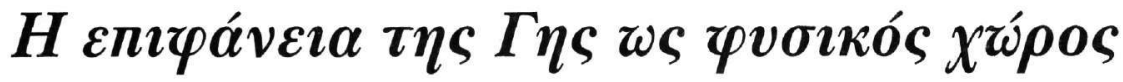

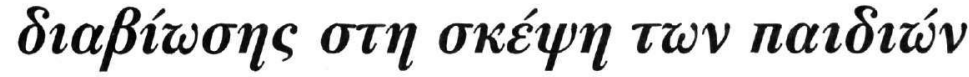

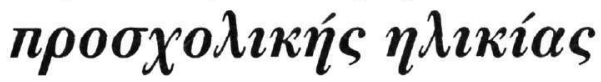

T

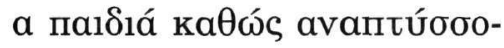

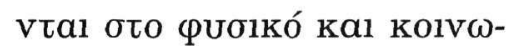

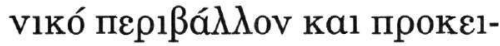

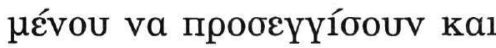
va katavońбouv autá поu oupßaí-

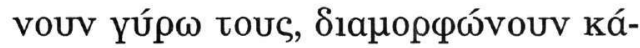

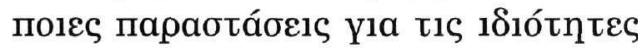

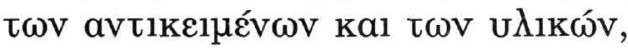

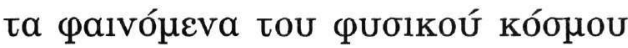

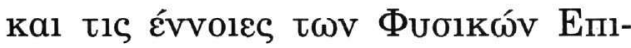

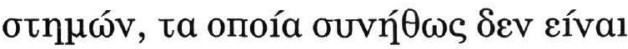

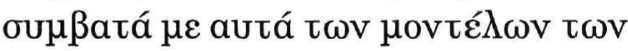

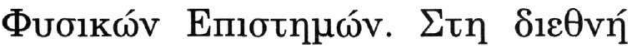

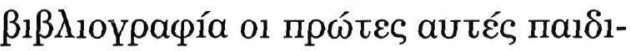

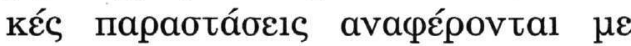

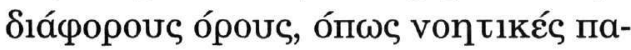

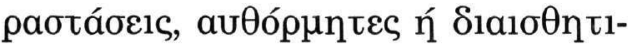

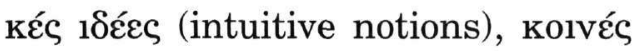

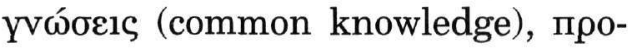
avıı

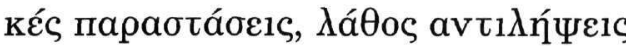

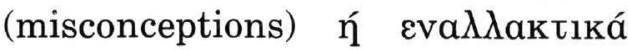
пגaíoıa (alternative frameworks).

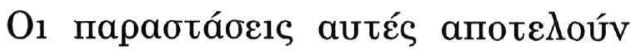

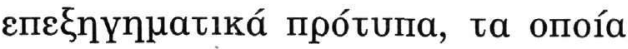

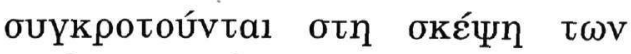

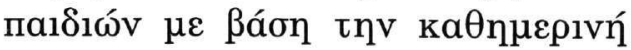

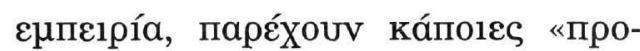

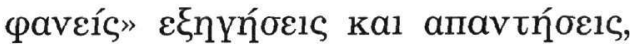

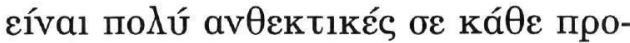

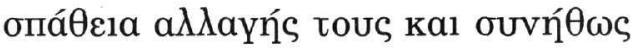

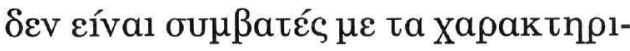

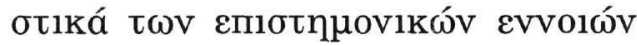
(Driver, Guesne \& Tiberghien, 1993. Fleer, 1995).

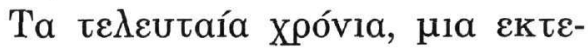

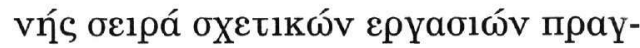

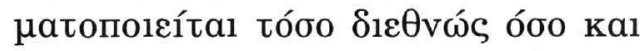

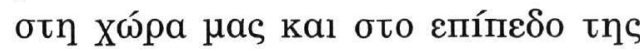

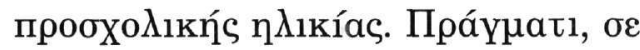

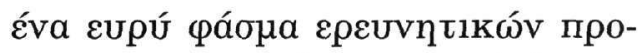

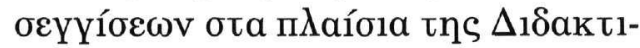

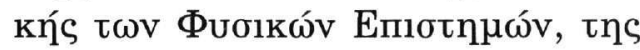

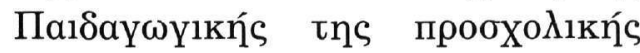

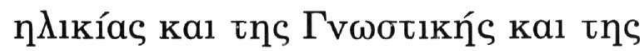

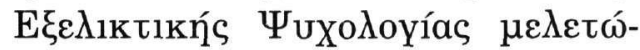

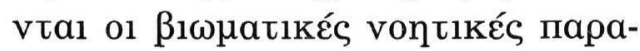

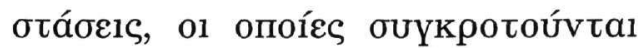

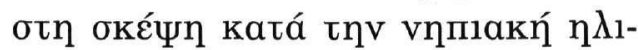

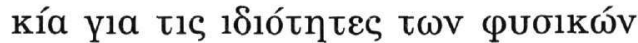

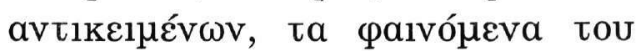

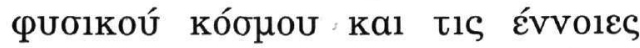

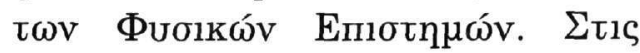

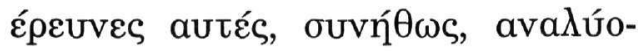

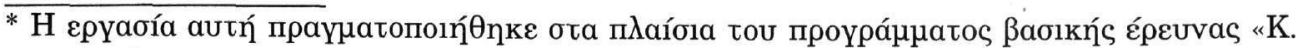

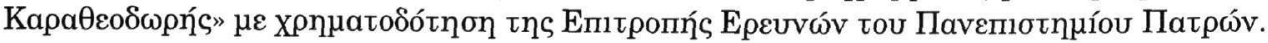




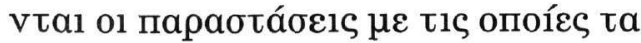

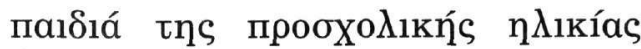

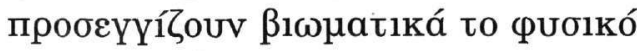

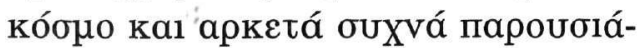

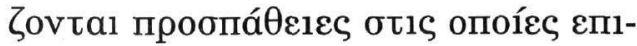

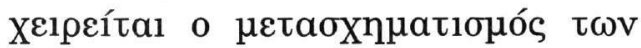

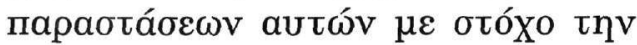

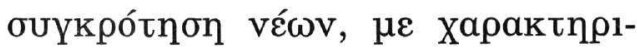

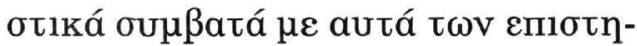

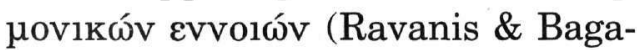
kis, 1998, Paßávns, 1999).

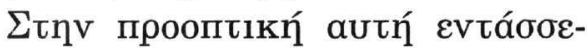

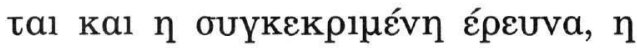

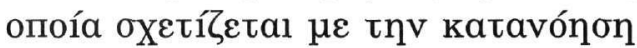

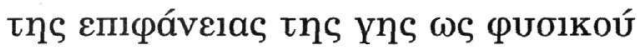

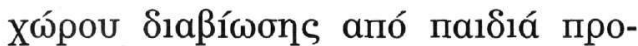

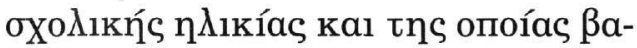

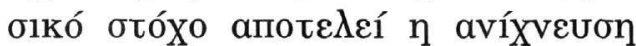

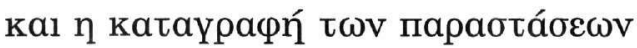

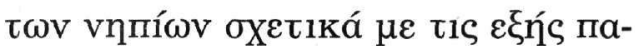

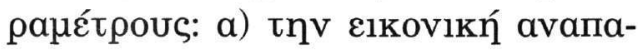

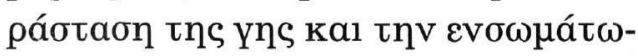

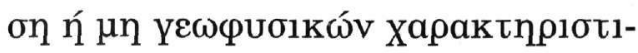

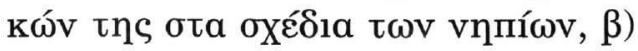

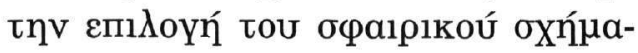

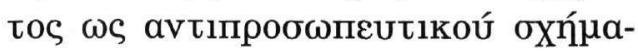

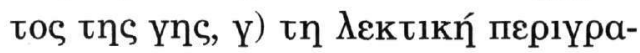
$\varphi \eta ́ ~ \gamma \varepsilon \omega \varphi$

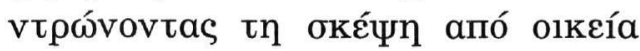

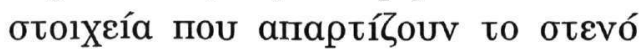

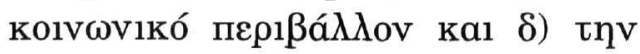
періурафи́ «апо́ коvта́» каl «апо́

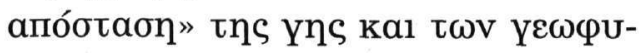

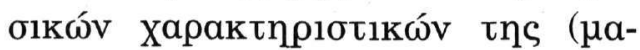

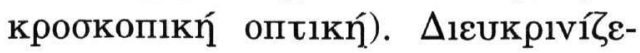

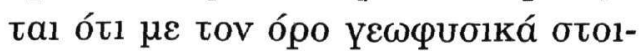

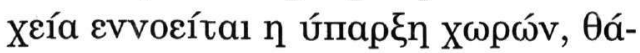

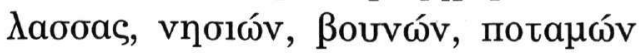

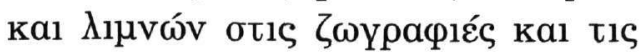

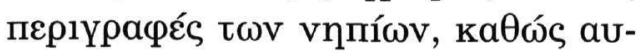

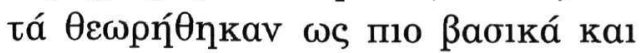

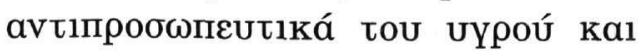

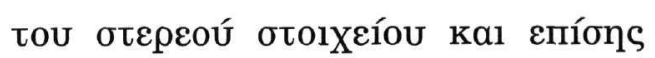

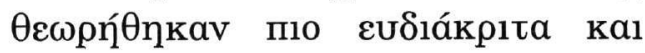

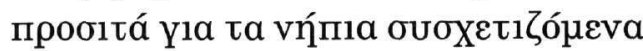

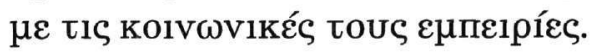

\section{H YП каı ta Yewpuoiká Xapa-

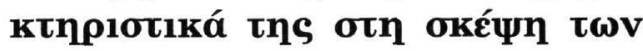

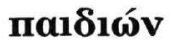

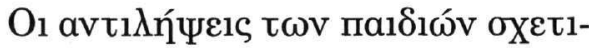

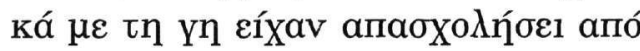
поגú vwpís tov Piaget (1929), о

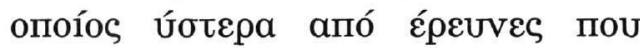

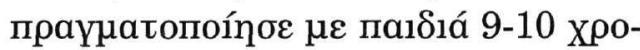

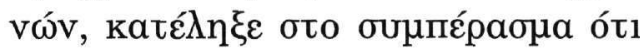

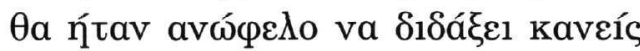

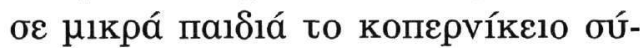

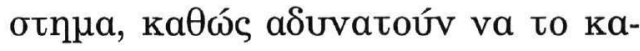
tavońfouv. Or Nussbaum \& Novak

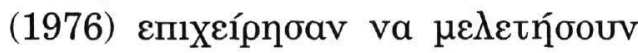

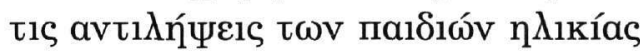

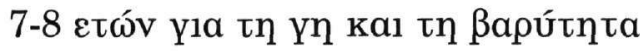

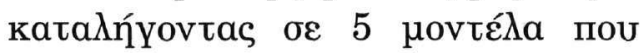

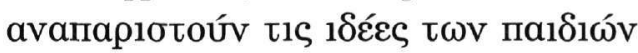

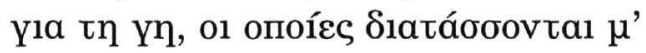

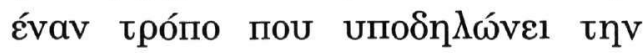

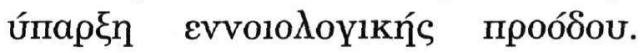

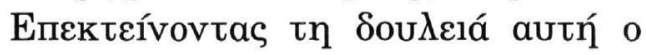

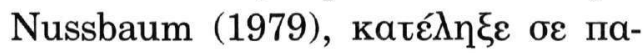

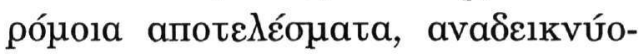

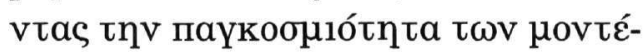

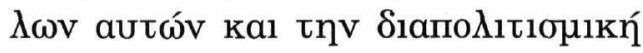

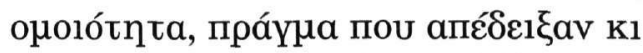

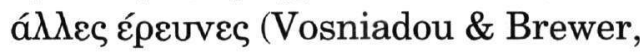

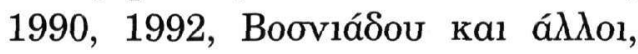
1996, Diakidoy, Vosniadou \& Hawks 1997, Baxter, 1995, Arnold, Sarge \&

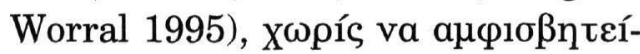

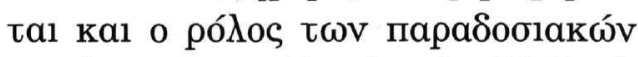

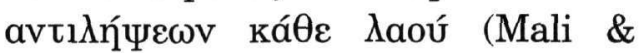
Howe 1979, Samarapungavan, Vos- 
niadou \& Brewer 1996). Гıа то $\varepsilon \lambda \lambda \eta$ -

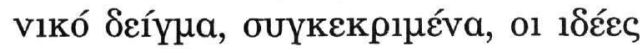

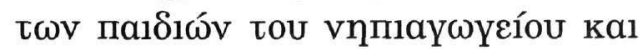

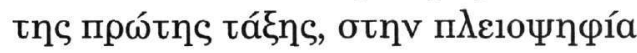

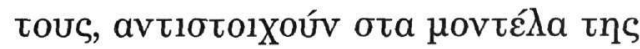

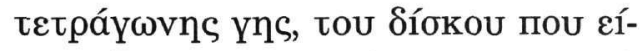

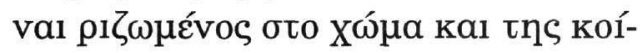

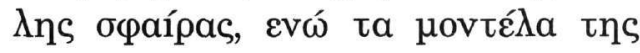

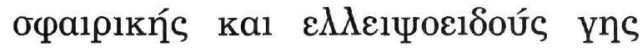

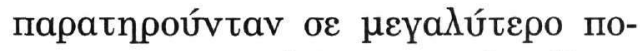

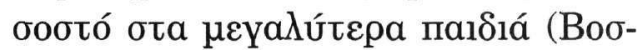

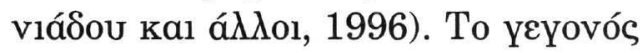

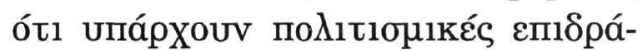

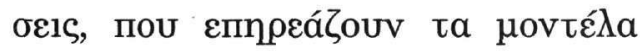

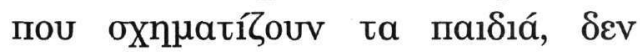

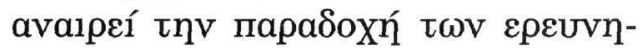

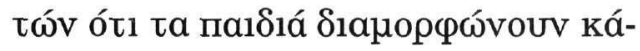

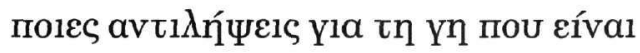
паүко́ориге.

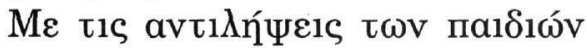

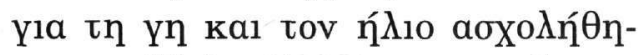
kav ๆ Klein (1982) kal ol Jones,

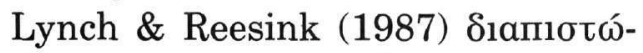

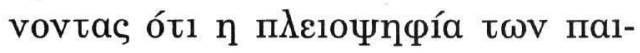

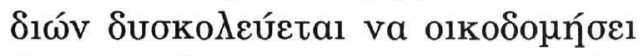

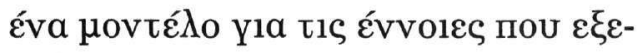

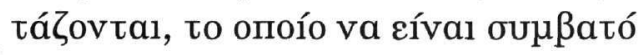

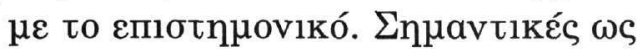

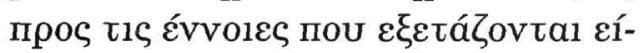
val kal ol ع́pєuveৎ tou Sharp (1995,

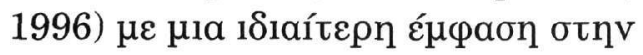

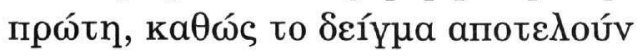

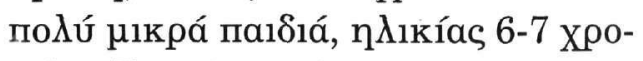

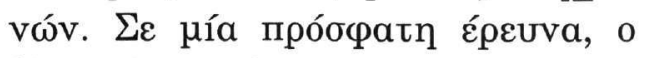

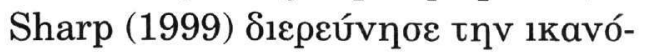

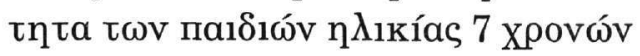

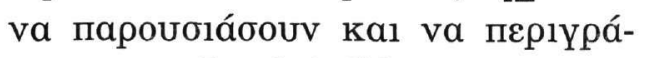

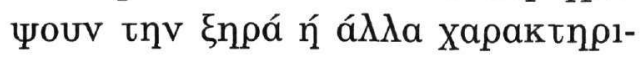

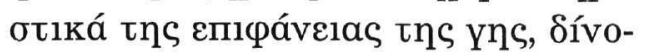

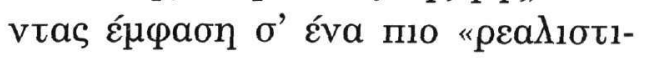

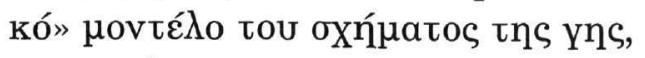

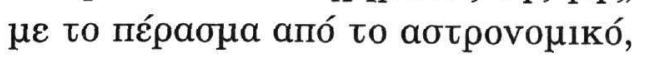

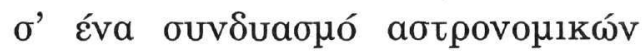

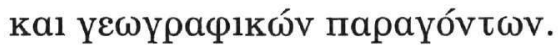

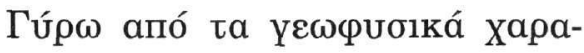

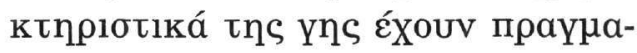

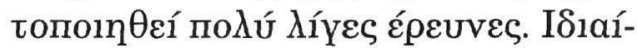

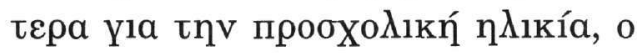

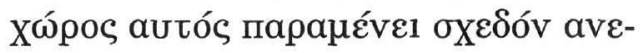

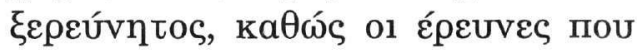

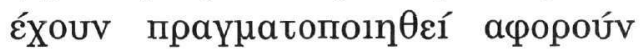

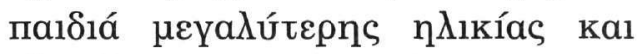

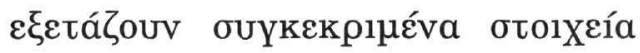

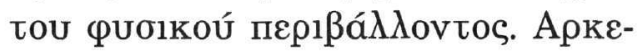

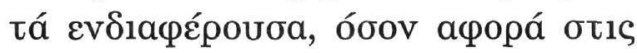

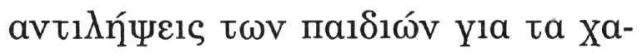

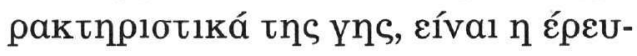
va $\tau \omega v$ Dove, Everett \& Preece

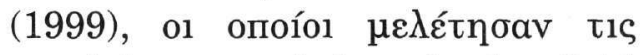

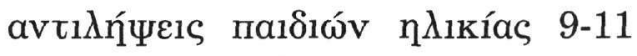

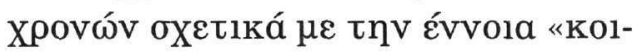

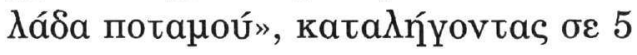

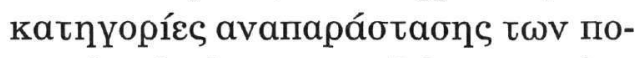

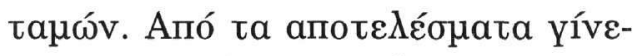

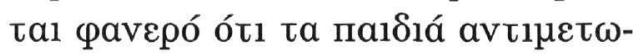

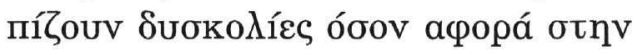

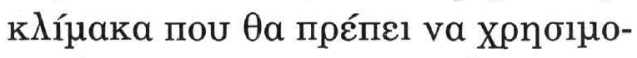

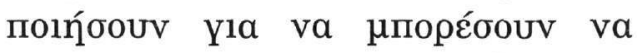

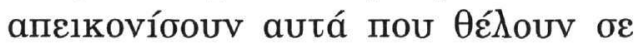

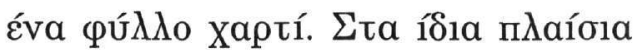

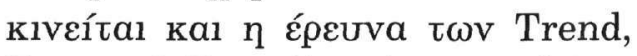
Everett \& Dove (2000), поч $\mu \varepsilon \lambda \varepsilon ́ t \eta-$

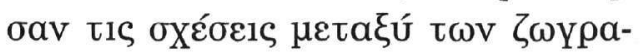

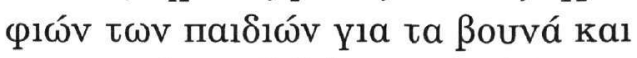

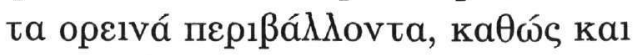

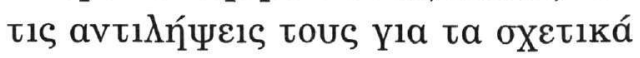

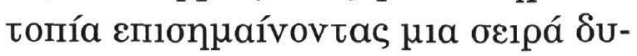

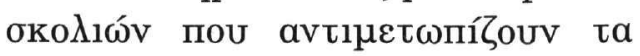

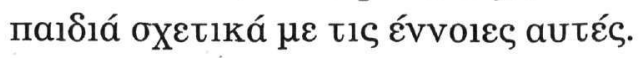

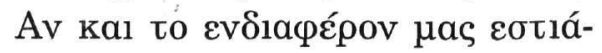

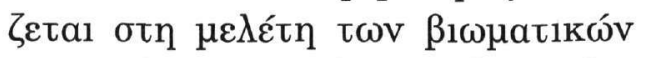

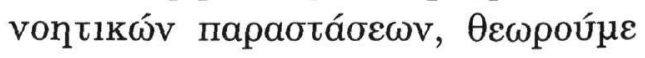

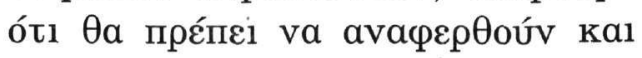

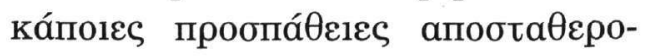


поínońs tous. O1 Nussbaum ka1

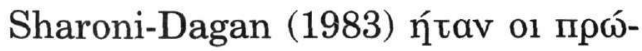

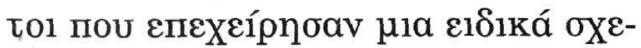

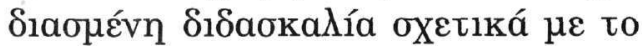
бхи́

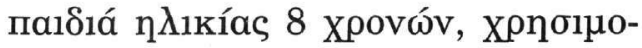

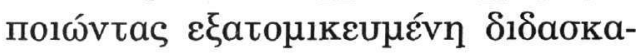

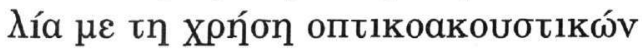

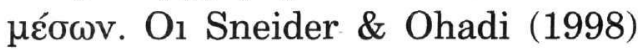

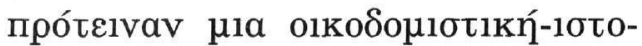

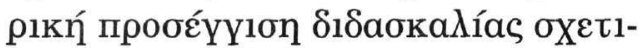

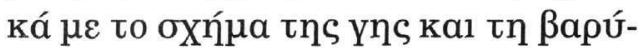

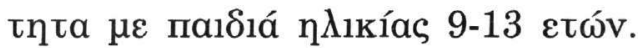
Oı Diakidoy, Kendeou (2001) єпıке-

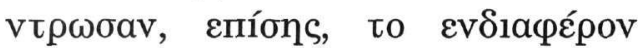

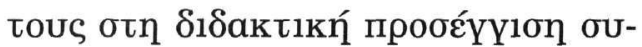

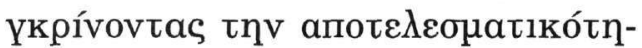

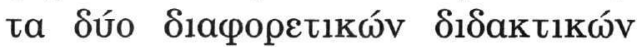

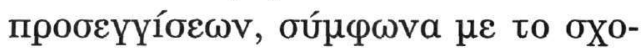

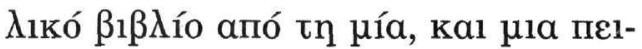

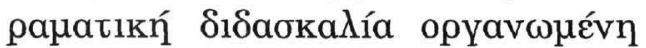

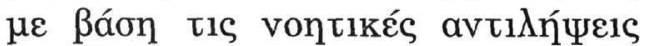

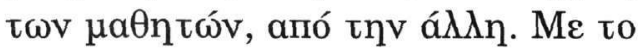

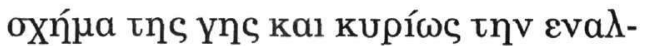

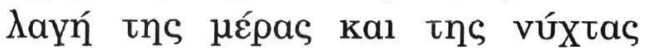
aoxoגńӨnkav kal or Valanides et al.

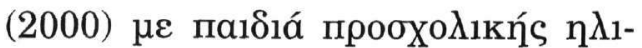
кías, ol опоі́ol пробпа́Өnбav va апо-

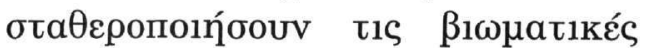

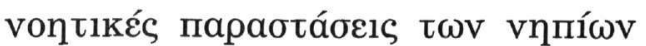

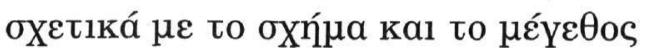

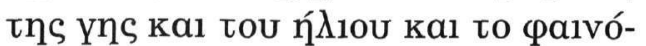

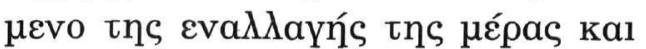
tᄁৎ vúX

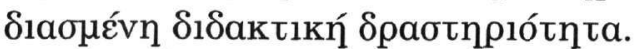

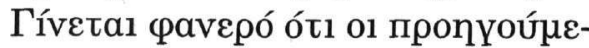

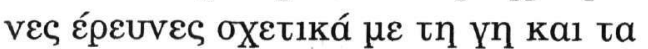

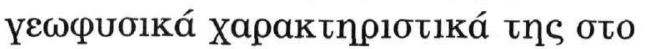

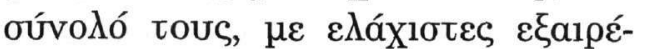

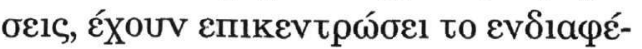

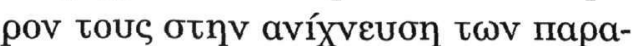

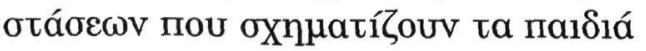

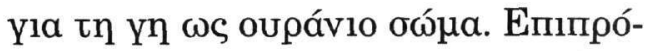

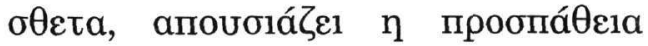

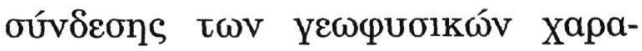

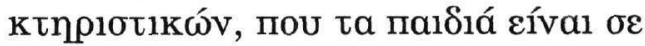

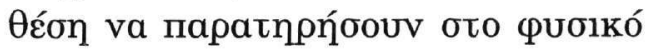

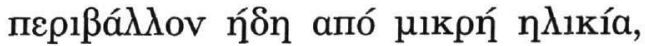

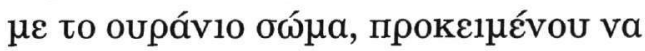

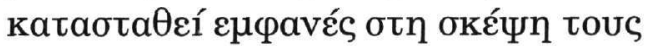

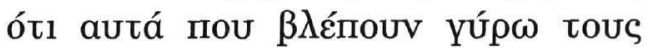

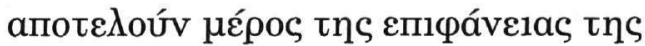

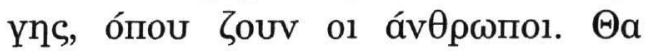

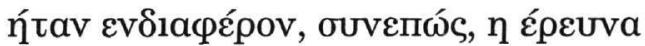

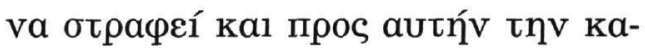

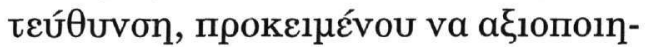

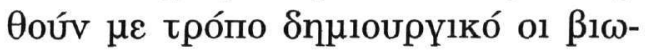

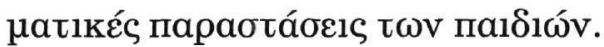

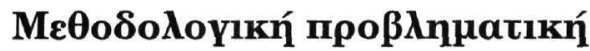

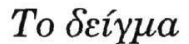

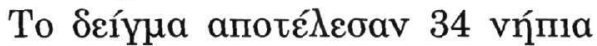

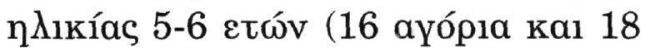

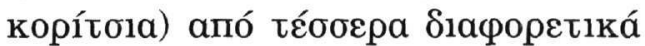

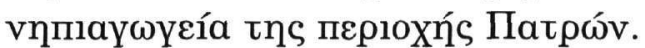

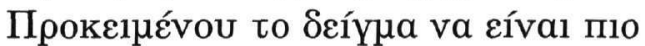

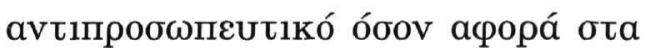

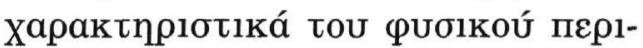

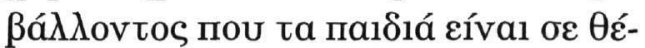

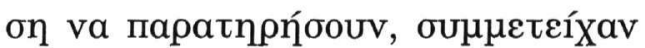
9 vท́mı aпó éva vппıүшүعío поч

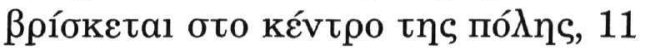

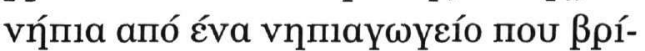

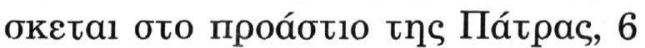

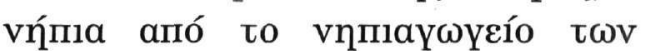

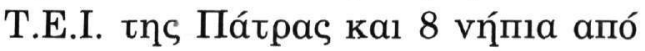

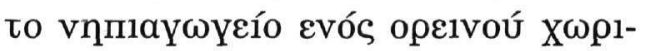

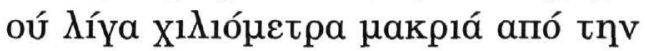

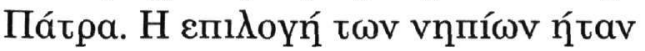

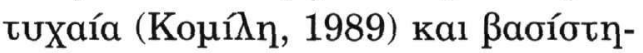

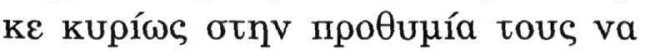

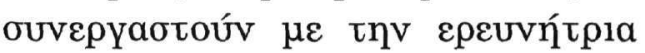




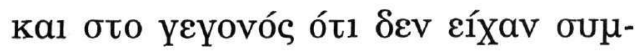

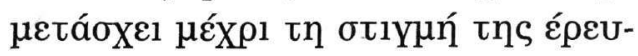

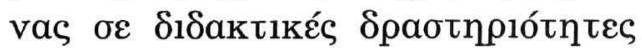

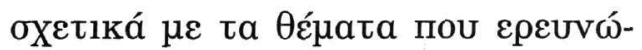

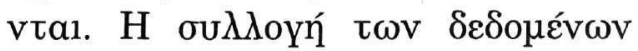

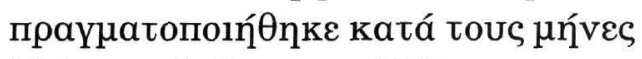
Máıo kaı Ioúvı tou 2000.

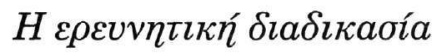

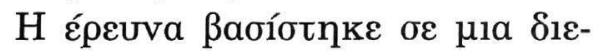

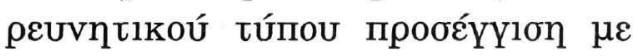

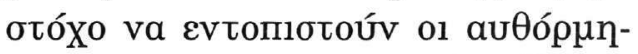

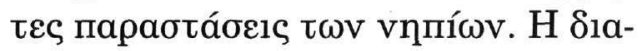

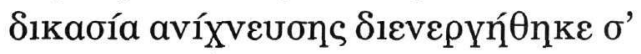

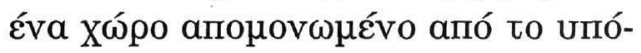

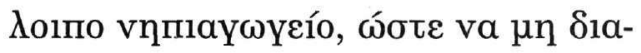

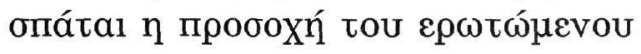

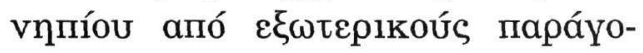

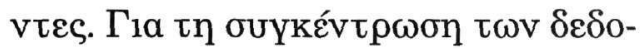

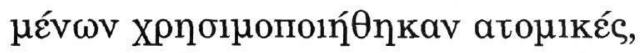

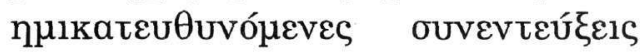

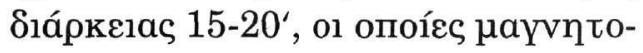

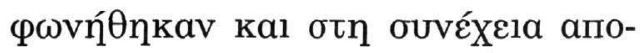

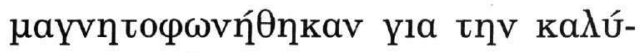

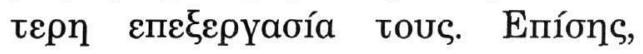

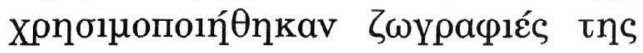

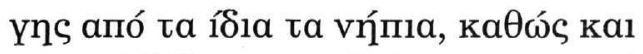

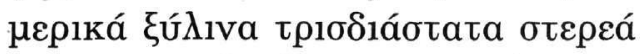

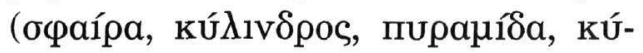

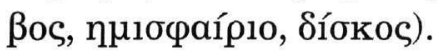

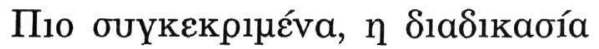

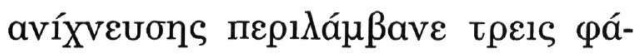

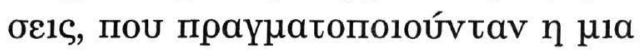

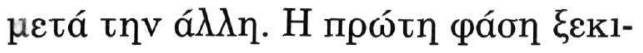

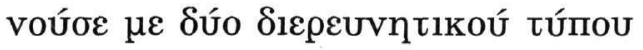

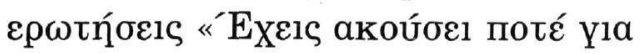

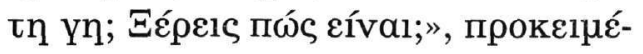

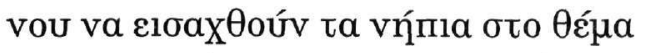

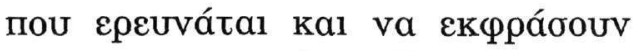

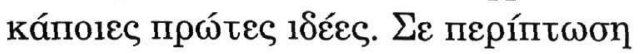

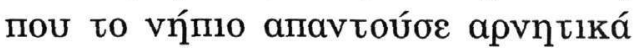

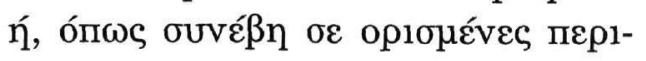

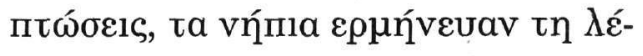

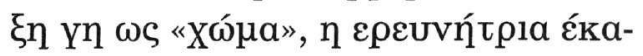

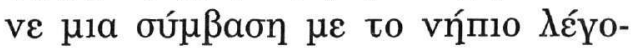

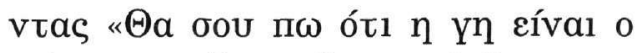

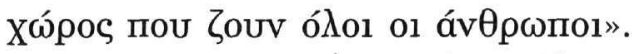

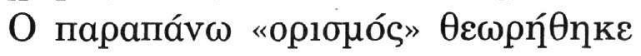

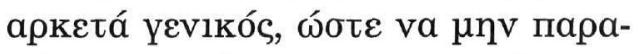

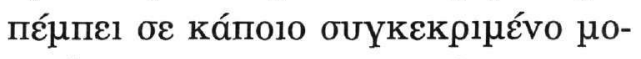

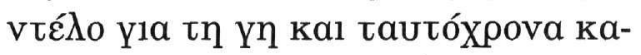

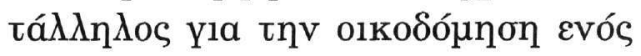

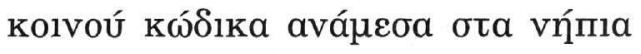

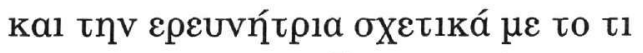

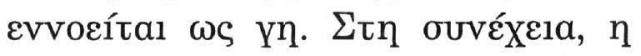

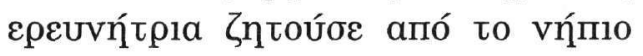

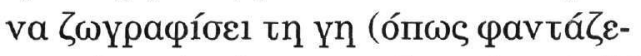

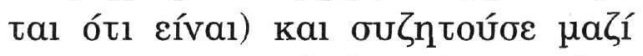

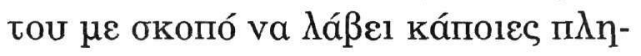

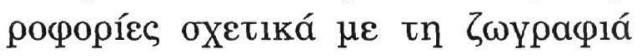

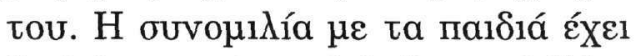

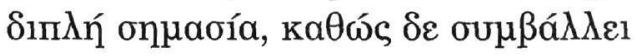

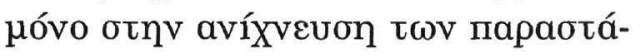

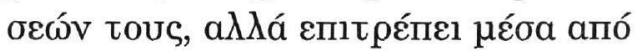

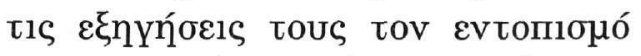

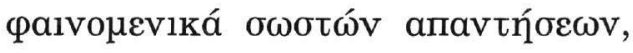

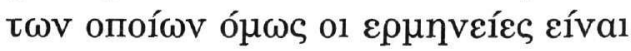

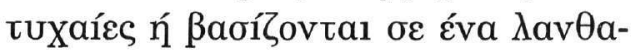

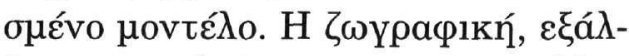

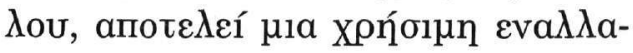

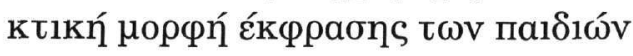

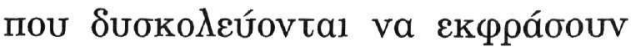

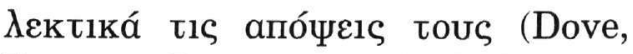
Everett, Preece, 1999). Béßaıa, $\eta$

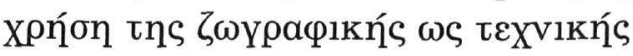

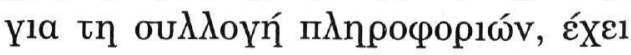

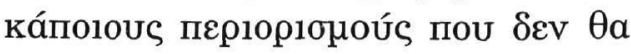

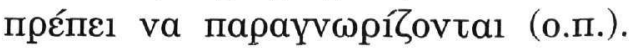

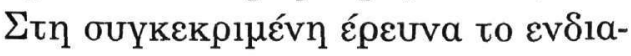

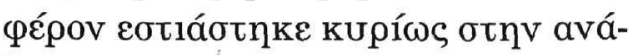

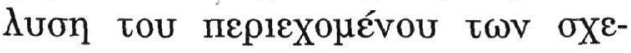

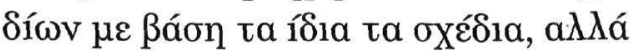

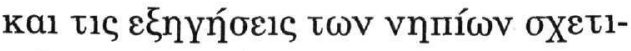

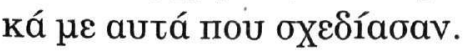




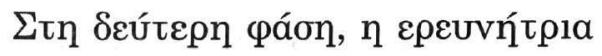

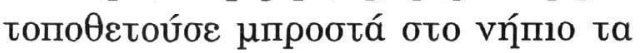

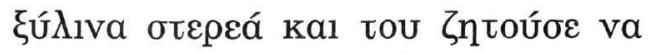

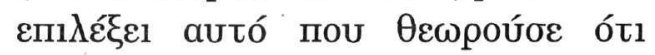

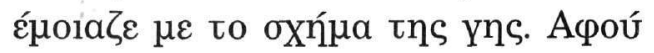

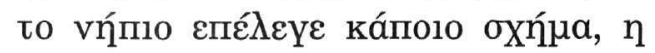

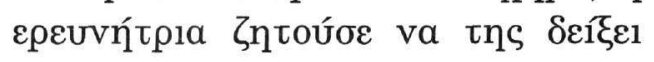

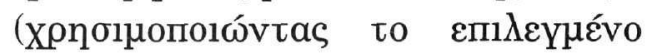

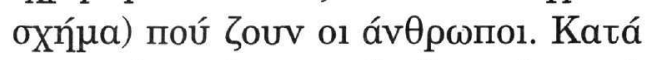

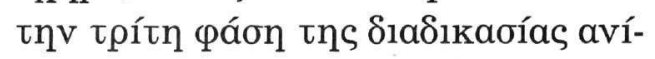

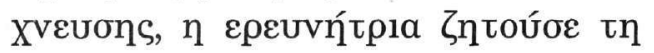

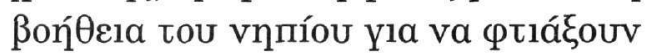

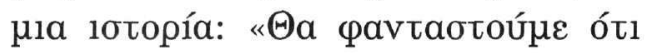

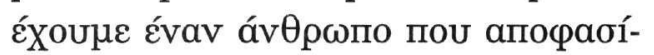

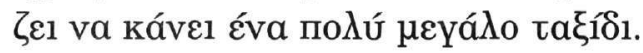

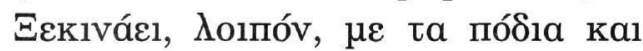

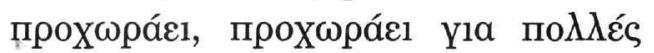

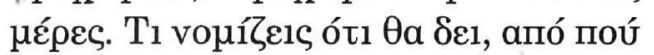

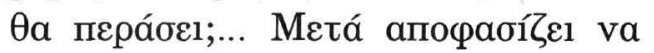

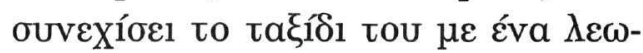

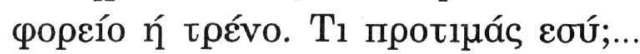

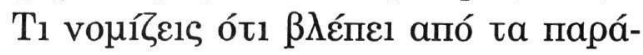

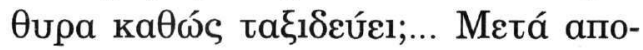

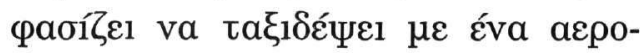

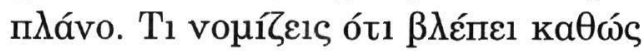

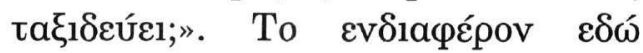

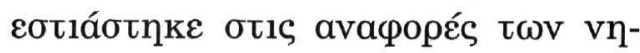

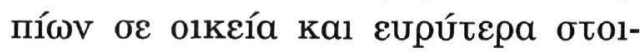

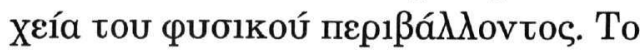

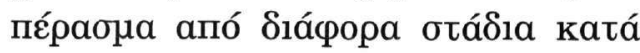

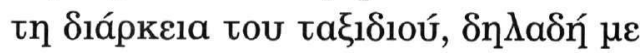

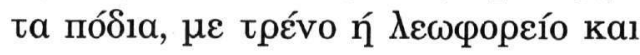

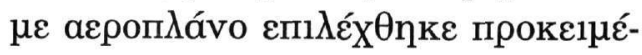

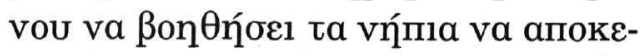
v

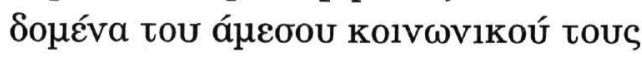

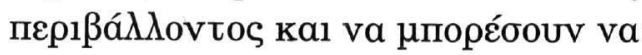

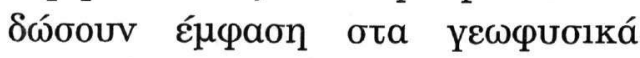

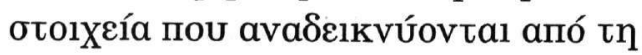

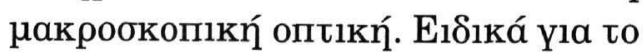

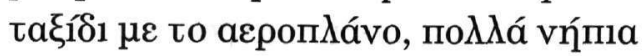

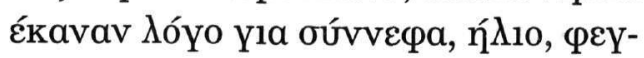

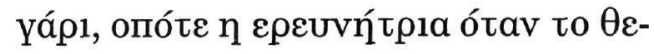

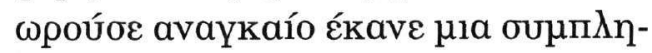

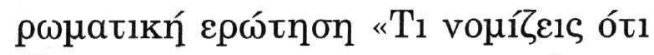

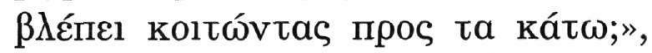

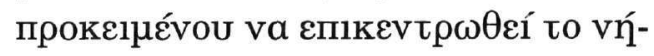

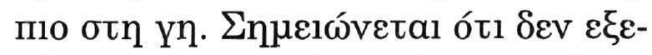

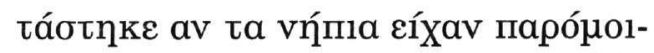

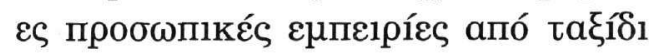

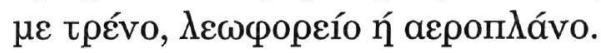

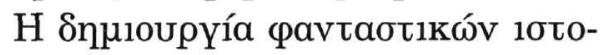

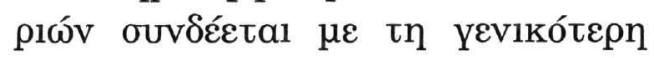

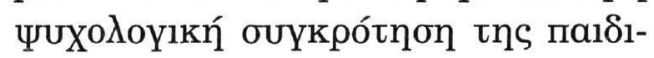

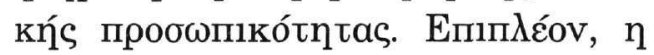

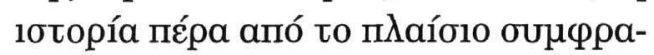

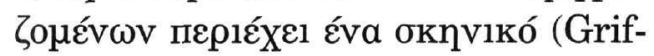
fiths \& Clyne, 1991, Rodari, 1985),

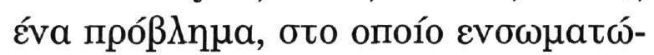

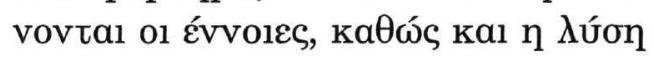

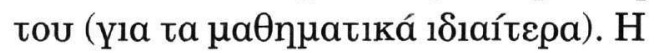

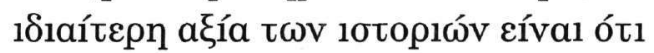

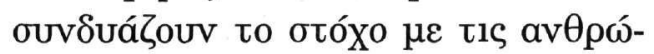

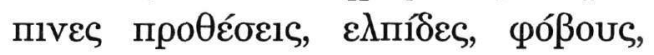

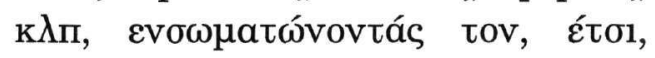

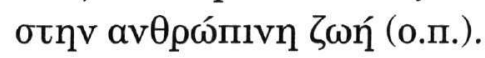

\section{Паpouoíaon

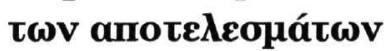

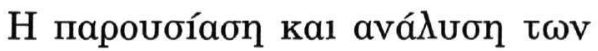

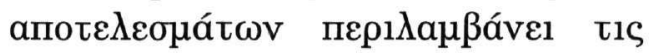

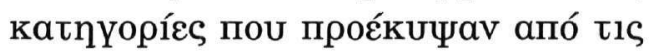

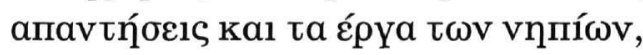

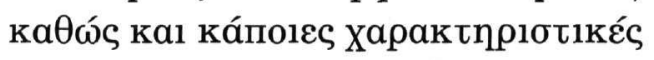

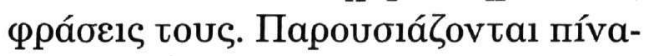

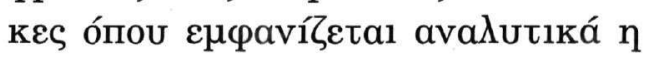

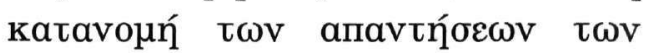

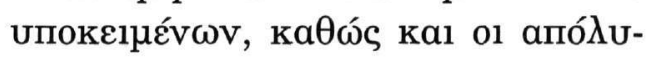

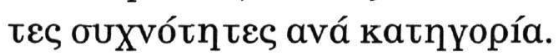

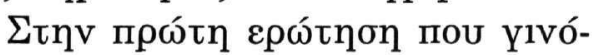

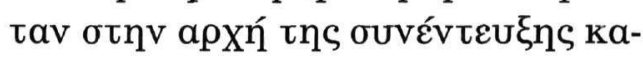

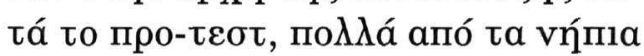




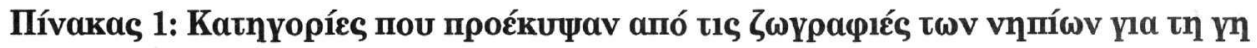

\begin{tabular}{|c|c|c|c|}
\hline КАTHГOPIE & YIOKEIMENA & $\mathbf{f}$ & $\%$ \\
\hline 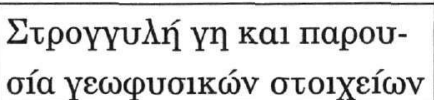 & $1,2,4,5,12,14,18,23$ & 8 & 23,5 \\
\hline 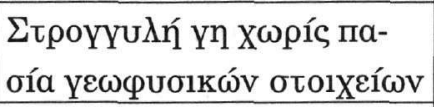 & $\begin{array}{l}7,10,11,13,17,19,22,24,25,26,28, \\
29\end{array}$ & 12 & 35,3 \\
\hline 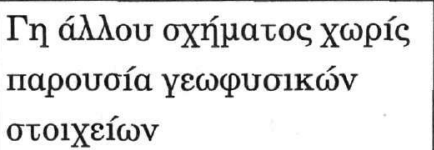 & $\begin{array}{l}3,6,8,9,15,16,20,21,27,30,31,32, \\
33,34\end{array}$ & 14 & 41,2 \\
\hline
\end{tabular}

$\mathrm{N}=34$

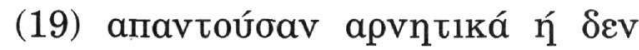

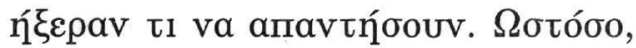

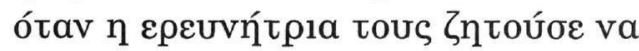

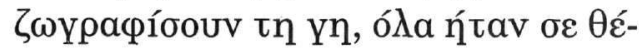

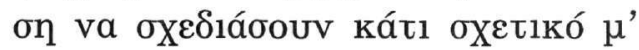

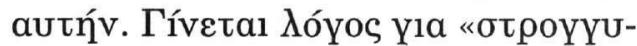

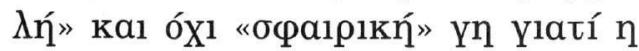

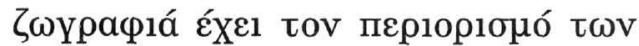

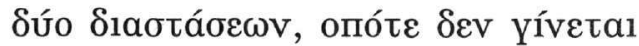

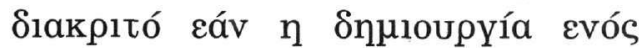

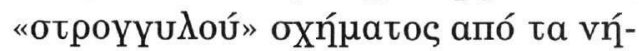

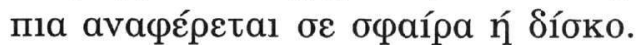

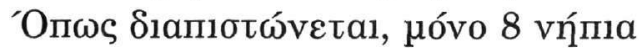

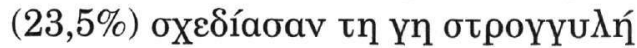

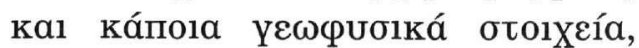

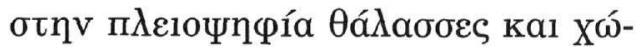

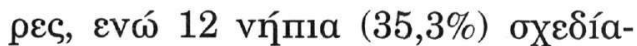

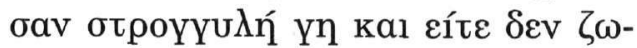

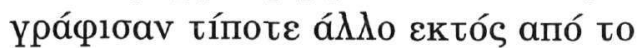

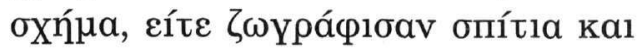

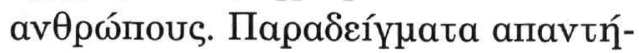

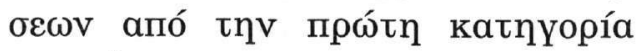

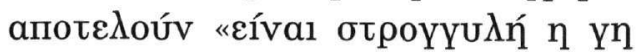

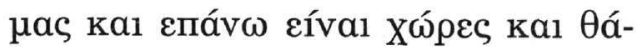

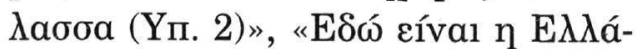

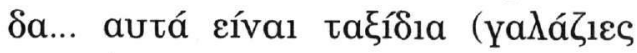

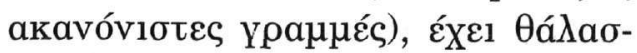

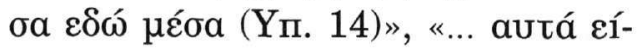

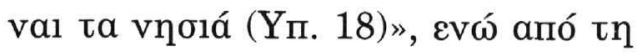

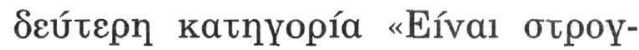

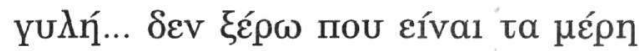

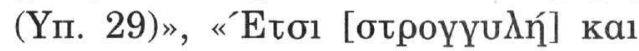

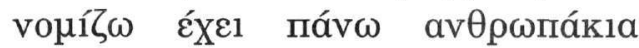

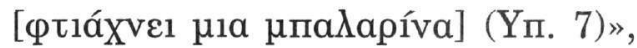

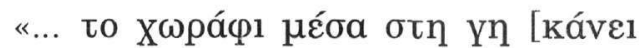

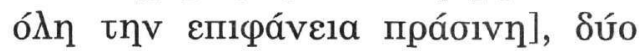

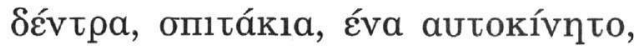

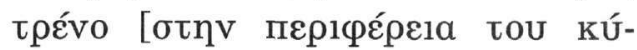

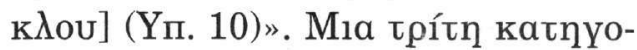
pía anotédeoar 14 vท́ma $(41,2 \%)$

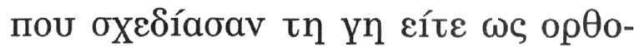

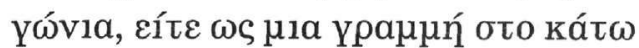

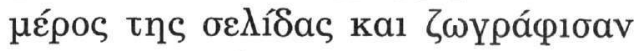

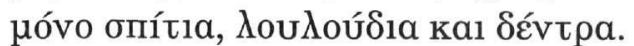

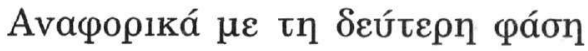

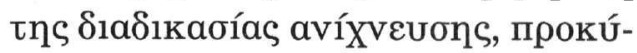

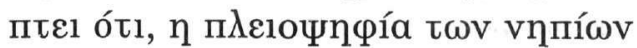

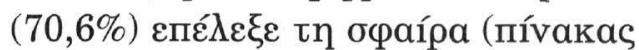

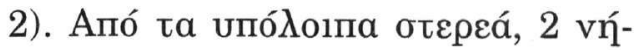

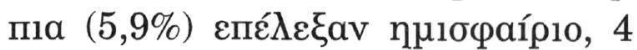

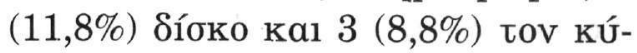

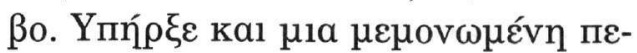

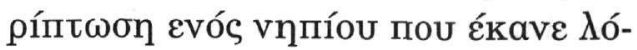

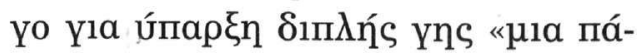

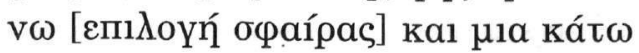

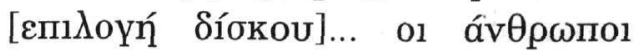

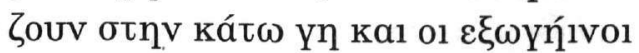

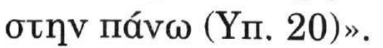




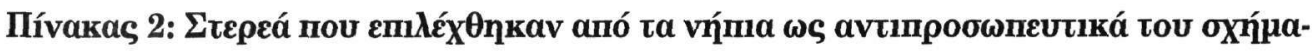
tos tns YMS

\begin{tabular}{|c|c|c|c|}
\hline 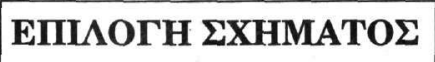 & YIIOKEIMENA & $\mathbf{f}$ & $\%$ \\
\hline \multirow[t]{2}{*}{$\Sigma$ ¿qípa } & $\begin{array}{l}1,2,5,7,10,11,12,13,14,16,18,19 \\
21,22,23,24,25,26,28,29,31,32,33\end{array}$ & 24 & 70,6 \\
\hline & 34 & & \\
\hline Ннıбчаípı & 15,30 & 2 & 5,9 \\
\hline$\Delta$ í́ros & $4,6,9,17$ & 4 & 11,8 \\
\hline Kúßos & $3,8,27$ & 3 & 8,8 \\
\hline$\Delta ı \lambda \eta ́$ үп & 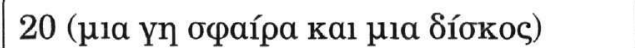 & 1 & 2,9 \\
\hline
\end{tabular}

$\mathrm{N}=34$

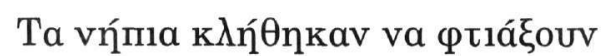

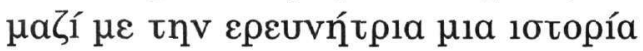

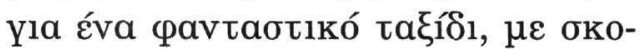

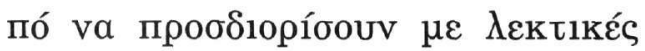

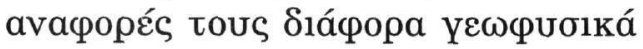

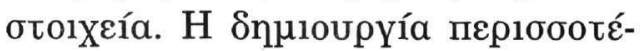

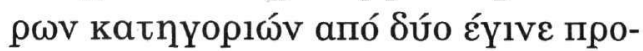

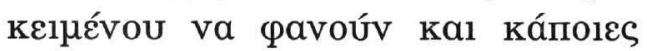

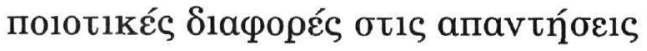

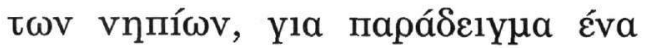

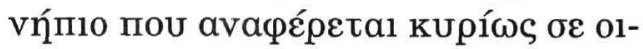

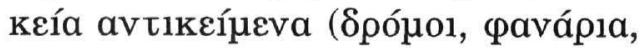

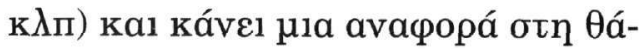

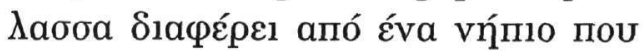

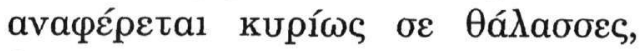

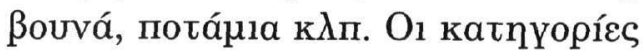

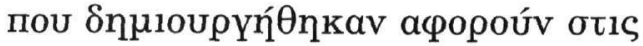

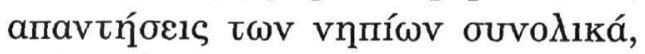

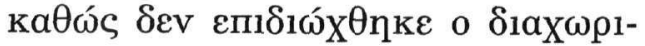

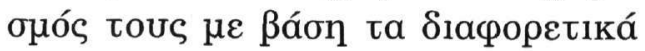

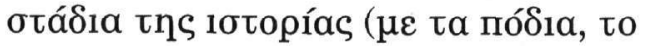

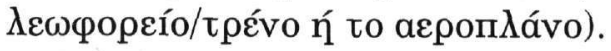

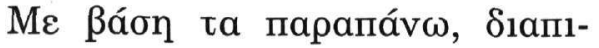

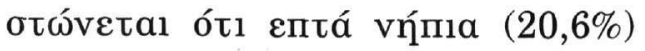

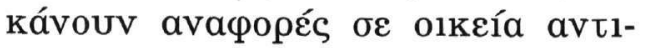

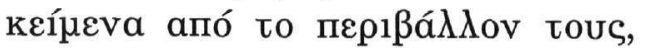

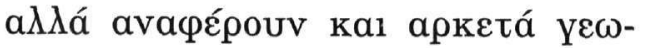

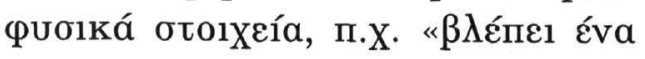

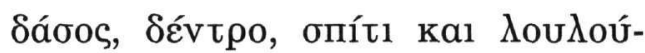

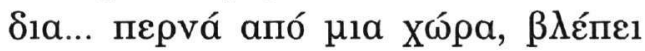

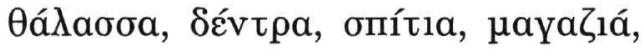

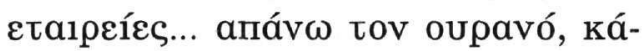

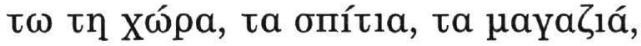

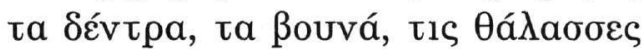

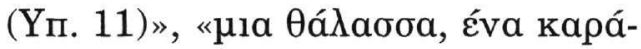

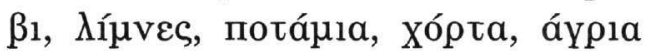
өnpía... бпítıa, подukatoikíes,

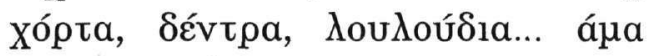

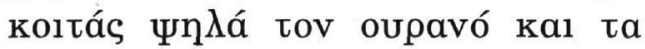

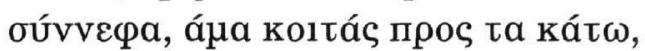

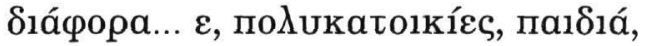

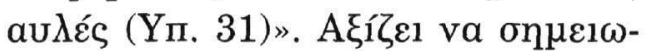

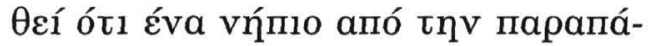

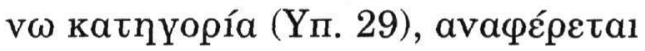

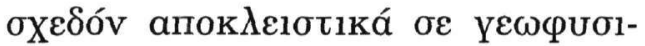

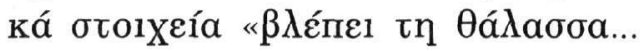

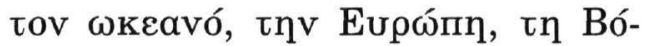

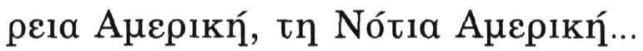

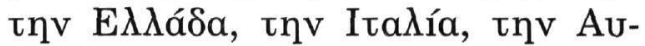

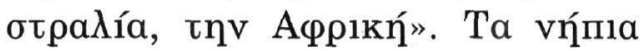

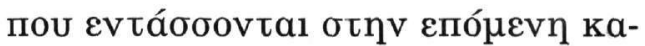

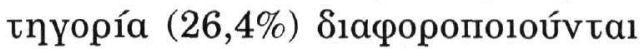

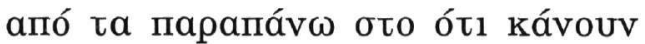

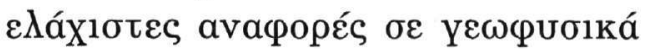

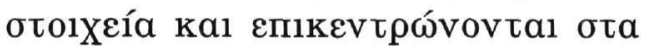

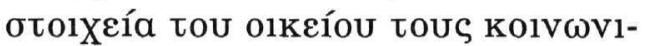




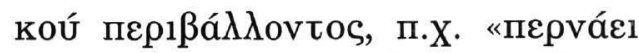

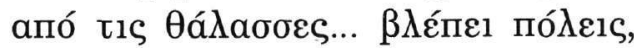

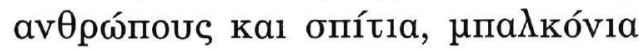

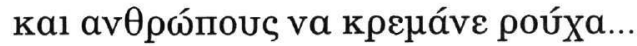

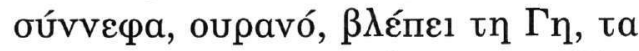

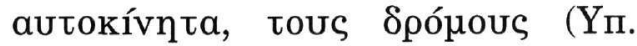

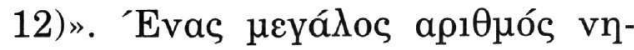

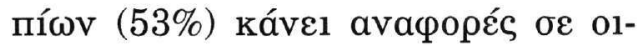

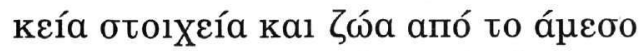

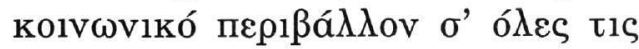

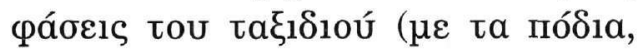

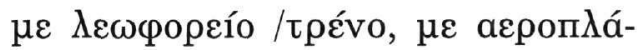

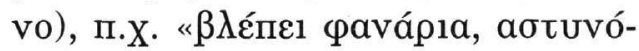

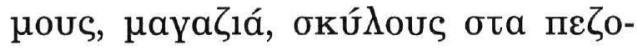

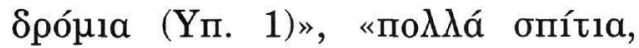

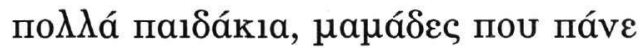

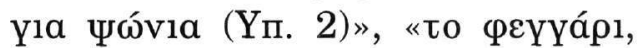

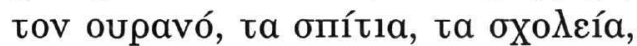

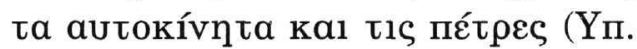

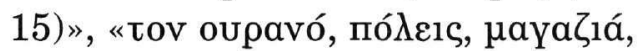

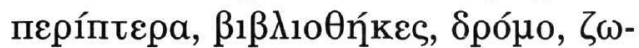

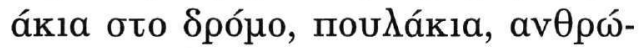

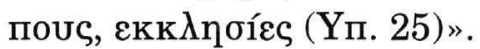

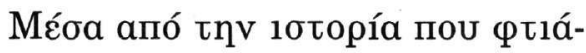

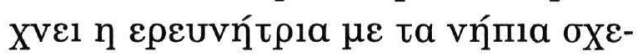

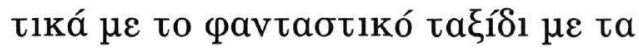

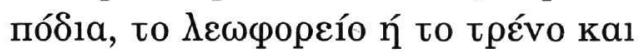

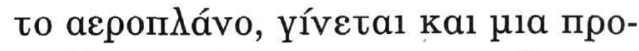

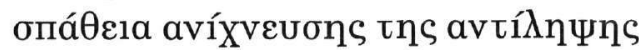

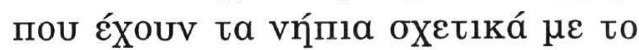

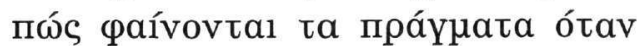

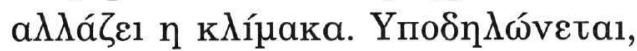

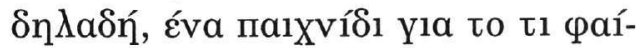

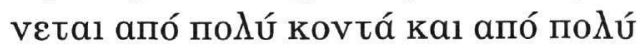

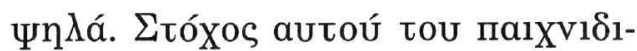

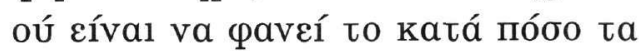

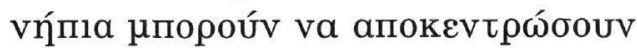

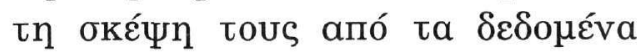

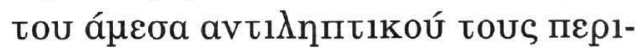

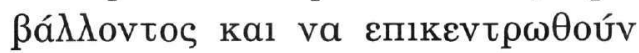

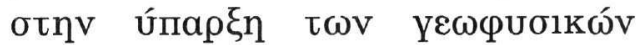

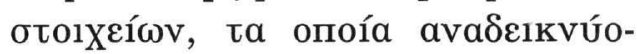
vtal kal Yívovtal mio epqavń ótav

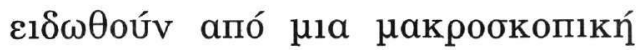
oпт1kń.

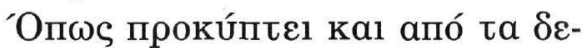

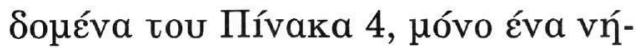

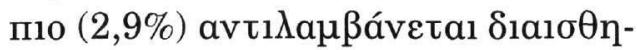

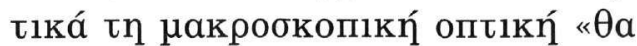

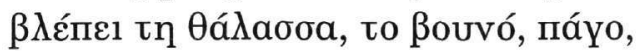

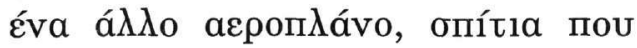

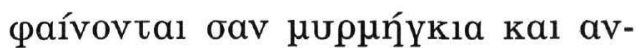

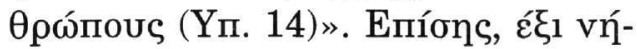

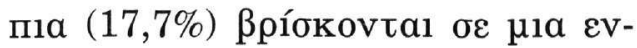

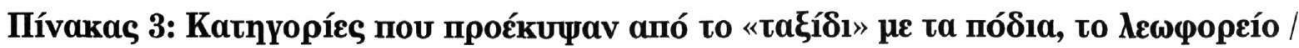
тре́vo ка1 to ąponגávo

\begin{tabular}{|c|c|c|c|}
\hline КАТНГОРІЕ & YIIOKEIMENA & $\mathbf{f}$ & $\%$ \\
\hline 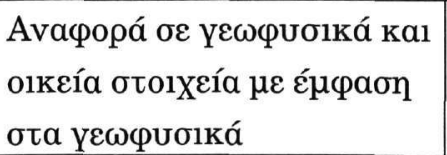 & $11,14,21,23,29,30,31$ & 7 & 20,6 \\
\hline 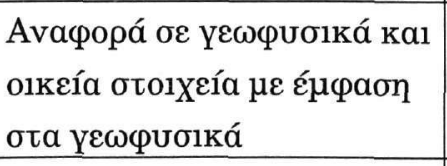 & $7,8,12,13,19,20,24,27,28$ & 9 & 26,4 \\
\hline 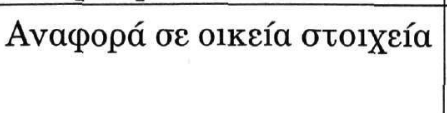 & $\begin{array}{l}1,2,3,4,5,6,9,10,15,16,17,18,22 \\
25,26,32,33,34\end{array}$ & 18 & 53 \\
\hline
\end{tabular}

$\mathrm{N}=34$ 


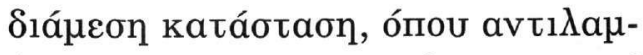

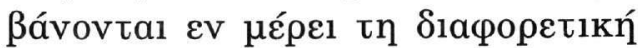

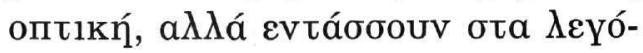

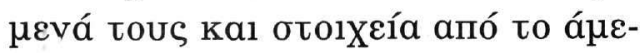

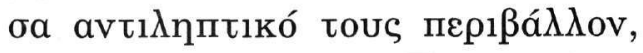

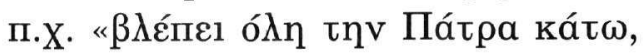

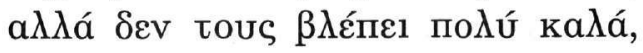

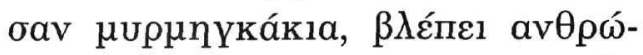

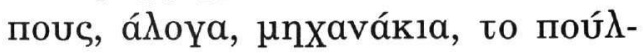

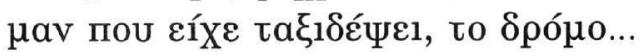

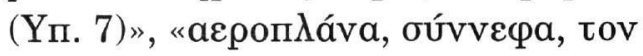

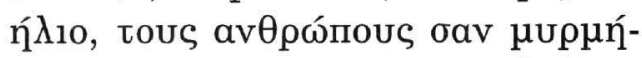

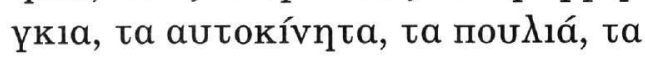

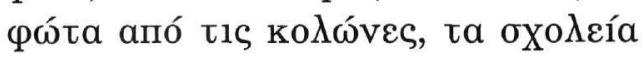

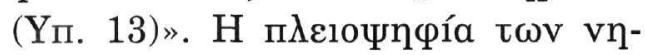

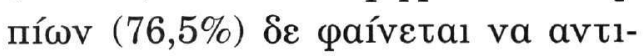

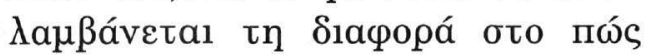

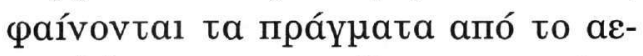

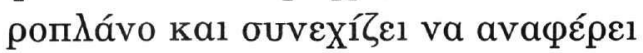

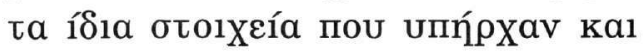

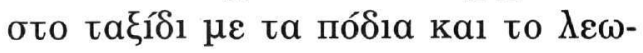

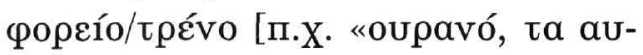

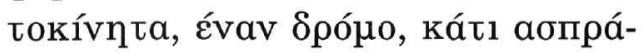

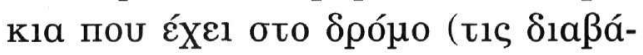

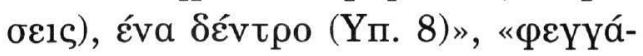

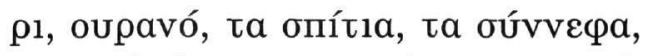

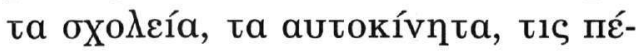

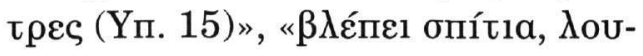

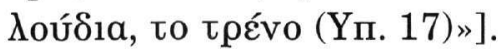

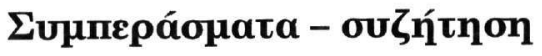

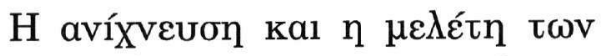

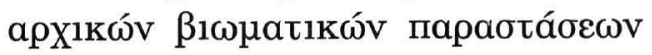

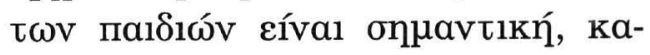

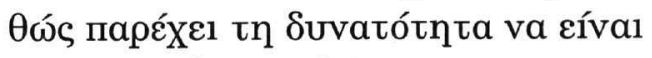

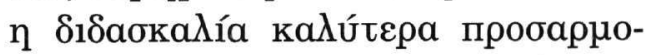

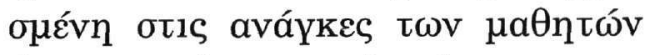
óoov a

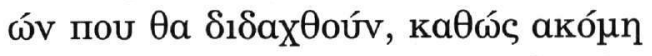

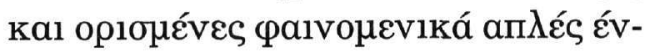

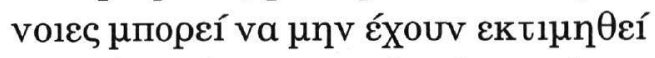

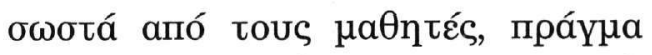

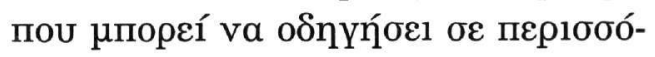

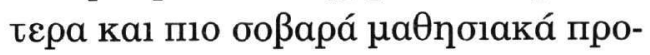

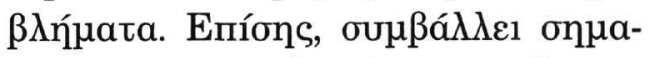

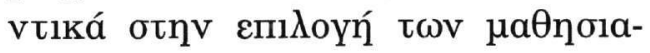

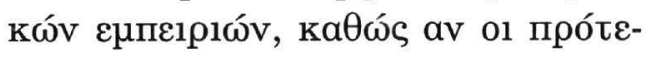

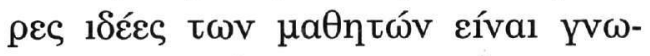

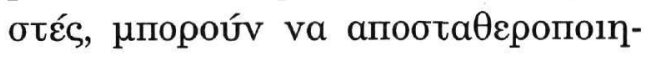

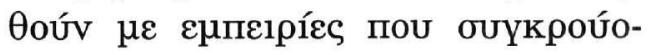

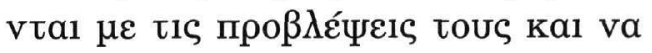

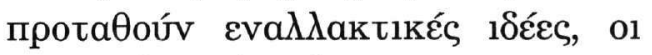

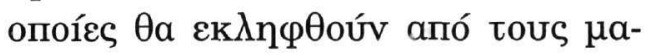

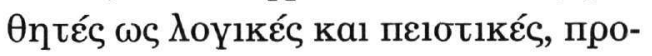

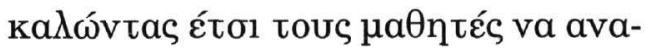

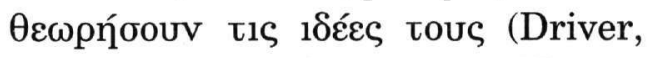

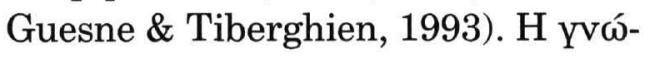

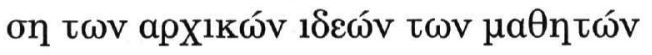

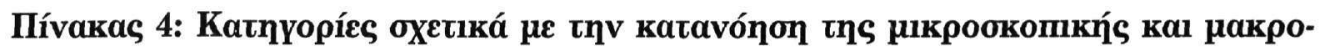

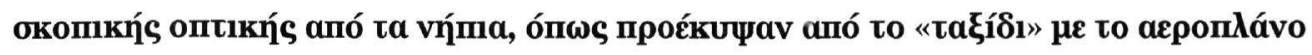

\begin{tabular}{|c|c|c|c|}
\hline КАТНГОРIE & YIIOKEIMENA & $\mathbf{f}$ & $\%$ \\
\hline 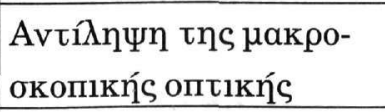 & 14 & 1 & 2,9 \\
\hline 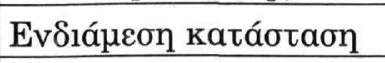 & $7,11,13,16,19,28$ & 6 & 17,7 \\
\hline 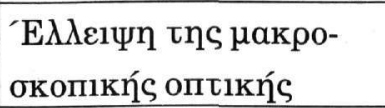 & $\begin{array}{l}1,2,3,4,5,6,8,9,10,12,15,17,18,20,21, \\
22,23,24,25,26,27,30,31,32,33,34\end{array}$ & 26 & 76,5 \\
\hline 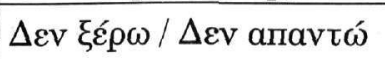 & 29 & 1 & 2,9 \\
\hline
\end{tabular}

$\mathrm{N}=34$ 


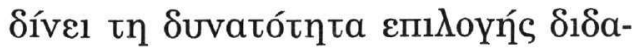

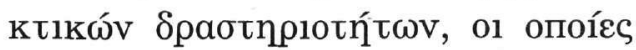

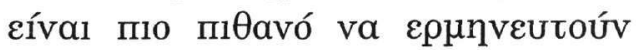

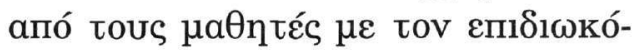
неvo тро́по.

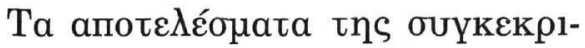

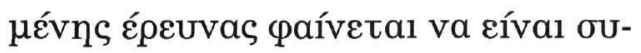

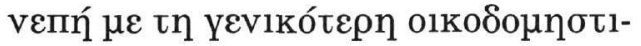

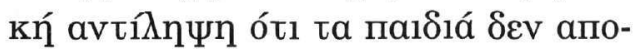

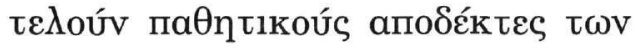

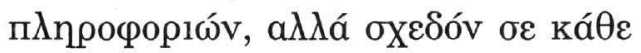

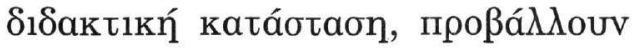

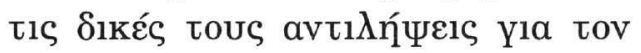

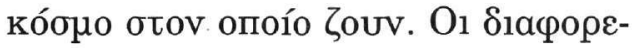

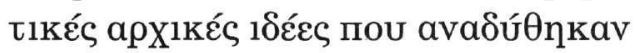

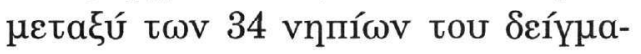

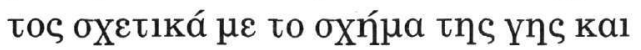

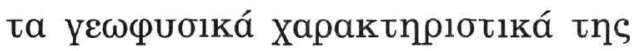

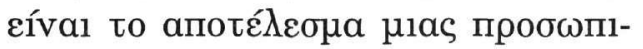

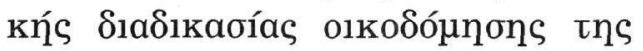

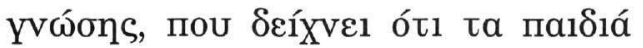

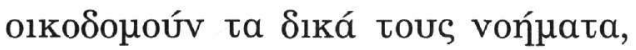

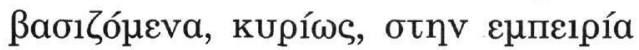

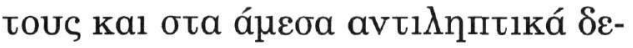

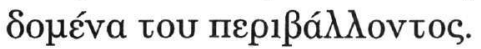

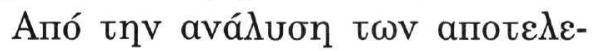

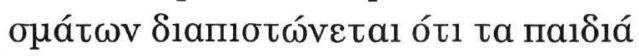

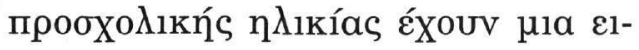

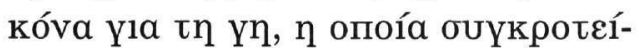

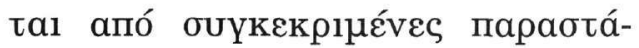

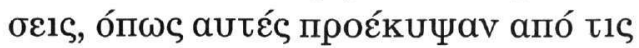

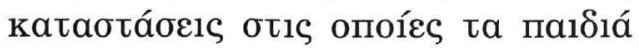

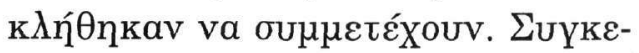

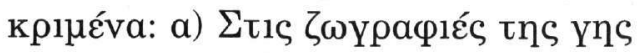

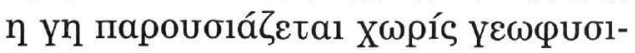

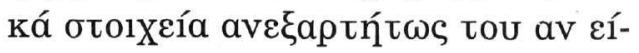

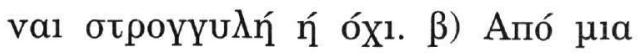

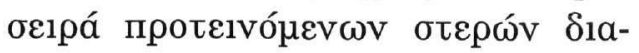

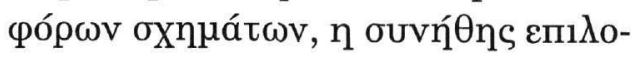

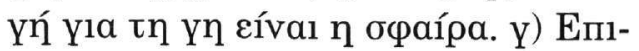

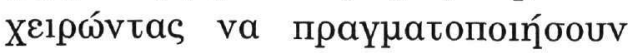

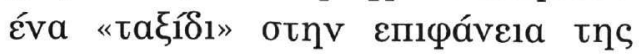

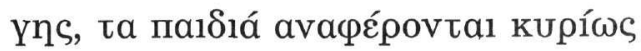
ota olkeía otolXeía tou kolvwvikoú

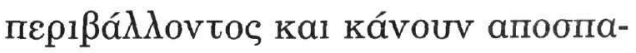

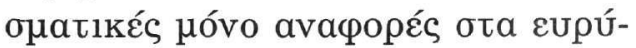

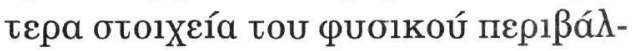

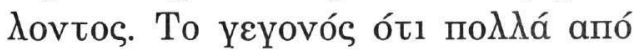

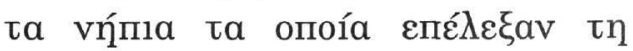

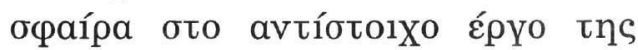

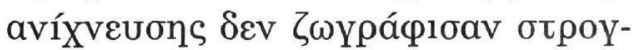

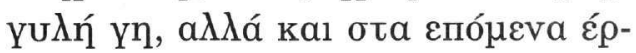

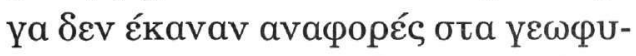

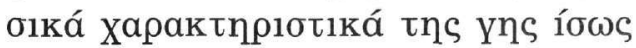

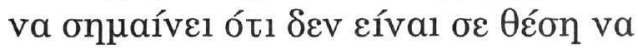

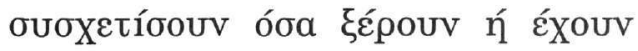

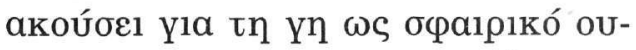

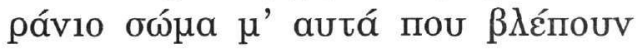

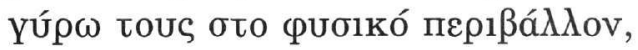

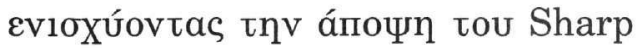

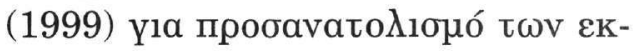

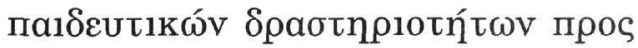

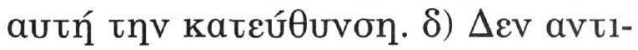

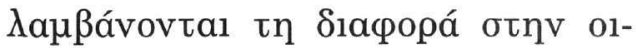

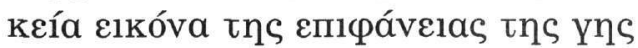

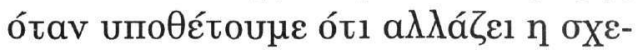

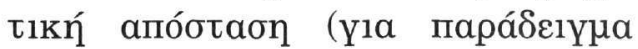

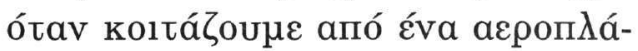

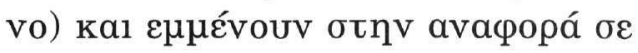

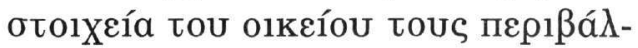
Aovtos. Sotóoo, to Yeyovós ót1

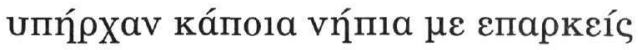

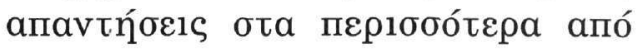

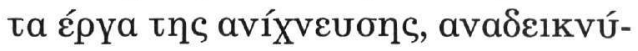

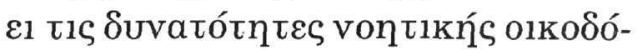

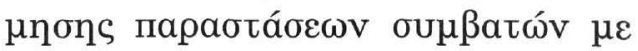

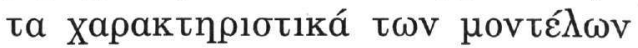

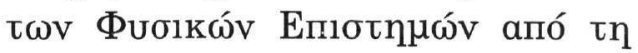

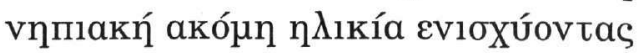

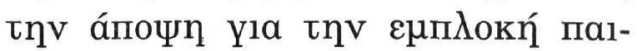

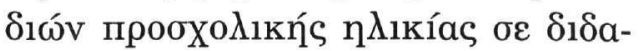

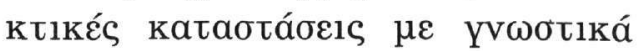

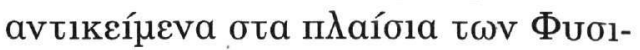

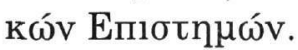




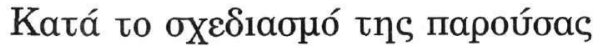

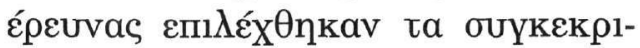

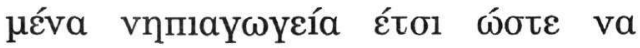

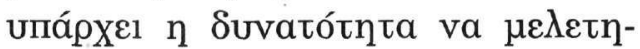

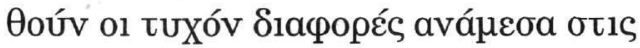

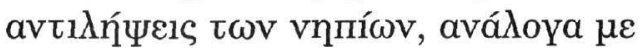

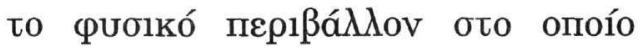

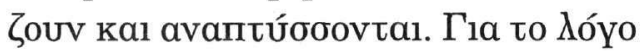

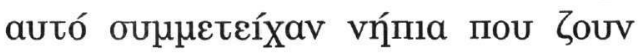

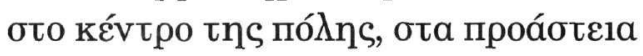

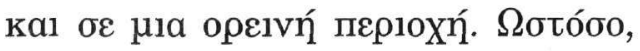

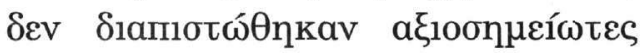

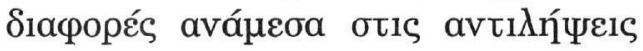

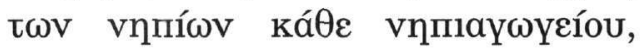

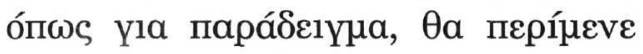

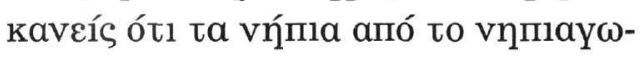

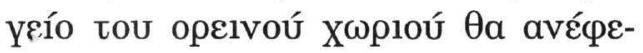

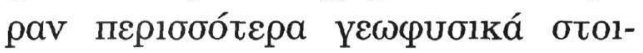

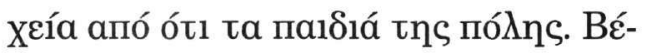

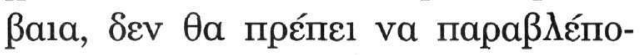

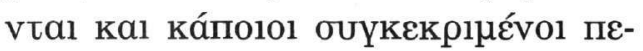

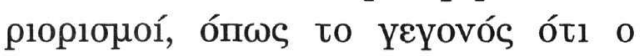

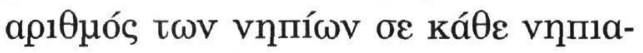
ү

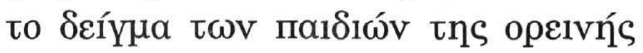

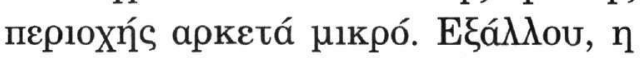

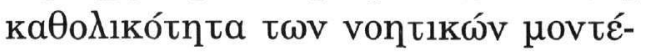

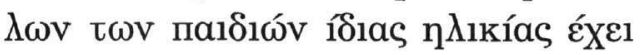

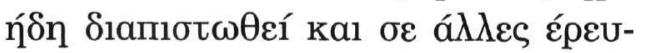
ves (Diakidoy, Vosniadou, Hawks,

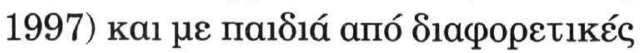

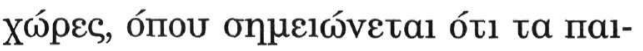

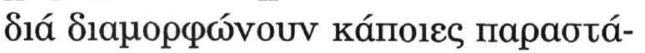

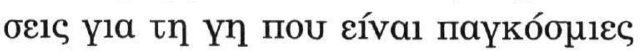
kal kolvés.

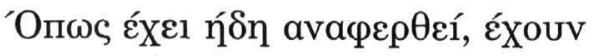

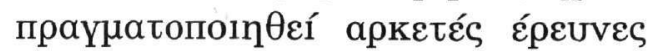

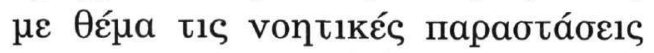

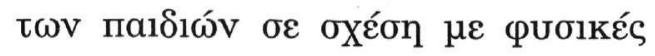

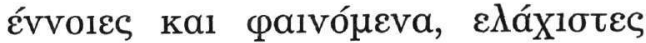

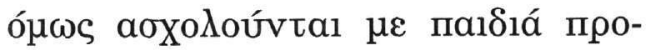

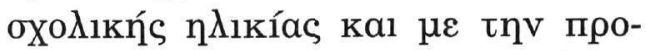

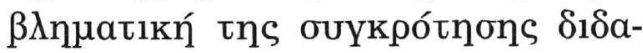

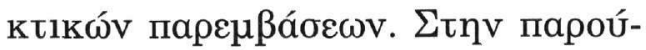

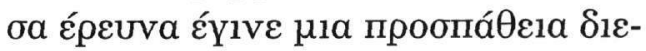

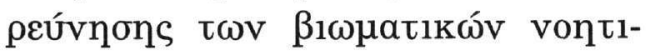

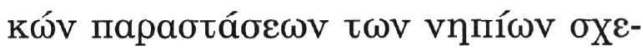

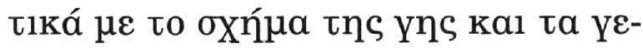

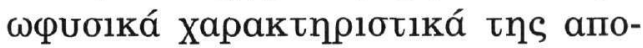

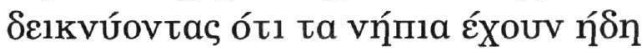

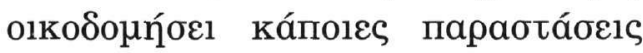

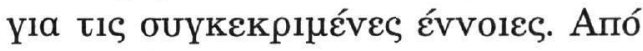

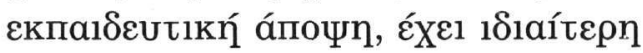

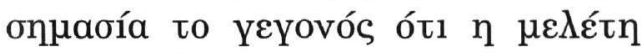

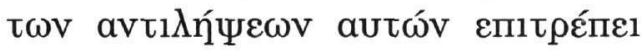

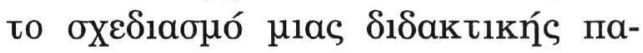

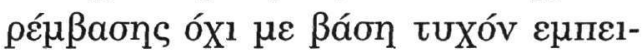

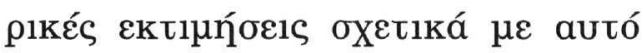

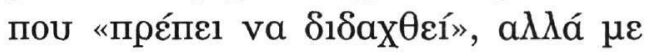

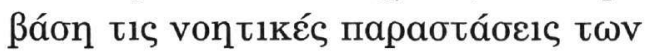

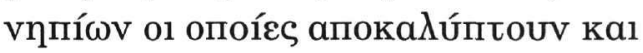

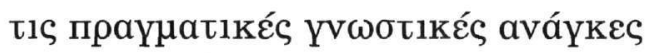

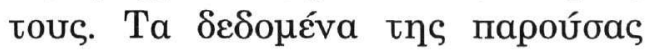

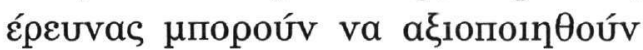

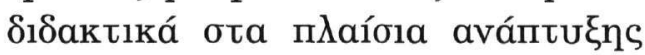

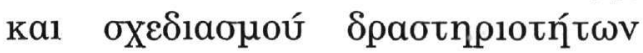

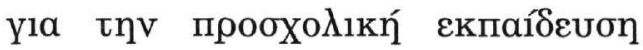

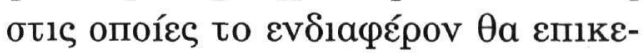

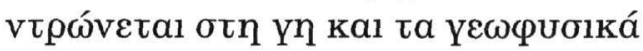

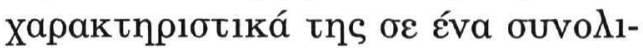

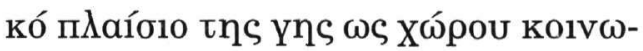

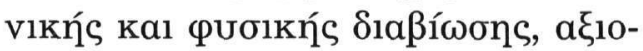

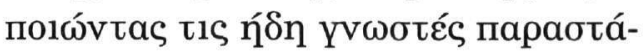

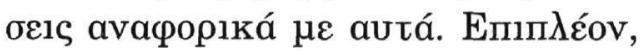

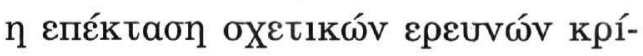

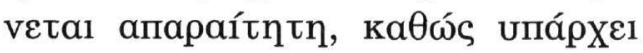

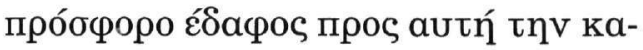

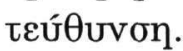




\section{Biß1oypaqía}

Arnold, P., Sarge, A., Worrall, L., (1995) Children's knowledge of the earth's shape and it's gravitational field. International Journal of Science Education, vol, 17, no 5, 635-641.

Baxter, J., (1995) Children's Understanding of Astronomy and Earth Sciences. In Glynn, S., M., Duit, R. (eds), Learning science in the schools, Mahwah, $\mathrm{NJ}$ : Elbaum.

Diakidou, I., A., Kendeou, P., (2001) Facilitating conceptual change in astronomy: a comparison of the effectiveness of two instructional approaches. Learning and Instruction, vol. 11, 1-20.

Diakidou, I., A., Vosniadou, S., Hawks, J., D., (1997) Conceptual change in astronomy: Models of the earth and of the day/night cycle in American-Indian children. European Journal of Psychology of Education, vol. 12, no 2, 159-184.

Dove, I., E., Everett, L., A., Preece, P., F., W., (1999) Exploring a hydrological concept through children's drawings. International Journal of Science Education, vol. 21, no 5, 485-497.

Driver, R., Guesne, E., Tiberghien, A., (1993) Or $\imath \delta \varepsilon ́ \varepsilon \varsigma ~ \tau \omega v ~ \pi a \imath \delta \imath \omega ́ v$

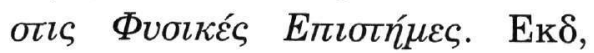

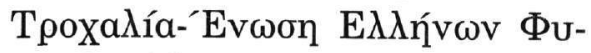

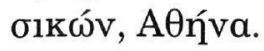

Fleer, M., (1995) The importance of conceptually focused teacher-child interaction in early childhood science learning. International
Journal of Science Education, vol. 17, no 3, 325-342.

Griffiths, R. \& Clyne, M., (1991) The power of story: Its role in learning Mathematics. Mathematics Teaching, vol. 135.

Jones, B., L., Lynch, P., P., Reesink, C., (1987) Children's conceptions of the earth, sun and moon. International Journal of Science Education, vol. 9, no 1, 43-53.

Klein, C., A., (1982) Children's Concepts of the Earth and the Sun: A Cross Cultural Study. Science Education, vol. 65, no 1, 95-107.

Mali, G. B., Howe, A., (1979) Development of Earth and gravity concepts among Nepali children. Science Education, 63 (5), 685-691.

Nussbaum, J, Novak, J. D. (1976) An Assessment of children's concepts of the Earth utilizing structured interviews. Science Education, 60 (4), 535-550.

Nussbaum, J. (1979) Children's conception of the Earth as a cosmic body: a cross-age study. Science Education, 63 (1), 83-93. Nussbaum, J., Sharoni-Dagan, N. (1983) Changes in second grade children's preconceptions about the earth as a cosmic body resulting from a short series of audio-tutorial lessons. Science Education, 67 (1), 99-114.

Piaget, J. (1929) The Child's Conception of the World, London: Routledge \& Kegan Paul.

Ravanis, K. \& Bagakis, G. (1998) Science Education in kinder- 
garten: sociocognitive perspective. International Journal of Early Years Education, 6(3), 315-327.

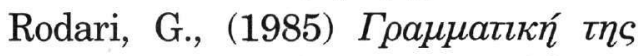

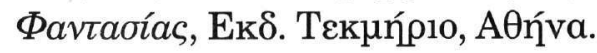
Samarapungavan, A., Vosniadou, S., Brewer, W., F., (1996) Mental models of the Earth, Sun, and Moon: Indian Children's Cosmologies. Cognitive Development, vol. 11, 491-521.

Sharp, G., J., (1995) Children's astronomy: implications for curriculum developments at Key Stage 1 and the Future of infant science in England and Wales. International Journal of Early Years Education, vol. 3, no 3, 17-49.

Sharp, G., J., (1996) Children's astronomical beliefs: A preliminary study of Year 6 children in southwest England. International Journal of Science Education, vol. 18, no 6, 685-712.

Sharp, G., J., (1999) Young Children's Ideas about the Earth in Space. International Journal of Early Years Education, vol. 7, no 2, 159-172.

Sneider, I., C., Ohadi, M., M., (1998) Unraveling Student's Misconceptions about the Earth's Shape and Gravity. Science Education, vol. 82, no 2, 265-284.

Trend, R., Everett, L. \& Dove, J., (2000) Interpreting Primary Children's Representations of Mountains and Mountainous Landscapes and Environments. Research in Science \& Technological Education, vol. 18, no 1, 85-112.
Valanides, N., Gritsi, F., Kampeza, M. \& Ravanis, K., (2000) Changing Pre-school Children's Conceptions of the Day/Night Cycle. International Journal of Early Years Education, vol. 8, no 1, 27-39.

Vosniadou, S., Brewer W.F., (1990) A cross-cultural investigation of children's conceptions about the earth, the sun and the moon: Greek and American data. Learning \& Instruction. European Research in an International Context. Analysis of Complex Skills and Complex Knowledge Domains. Mandl, H., De Corte, E., Bennett, N., \& Friedrich, H.F. (eds), v.2:2, pp. 605-629, Oxford: Pergamon.

Vosniadou, S., Brewer, W., F., (1992) Mental models of the Earth: A Study of Conceptual Change in Childhood. Cognitive Psychology, vol. 24, 535-585.

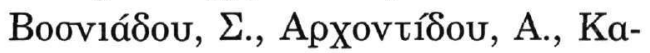

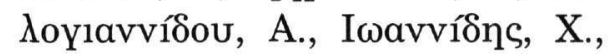

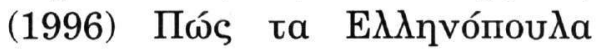

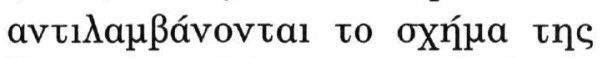

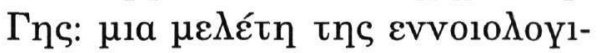

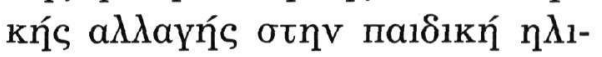

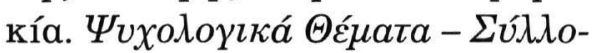

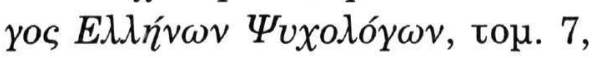
זعUX. 1, 30-51.

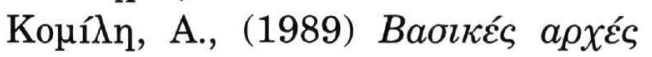

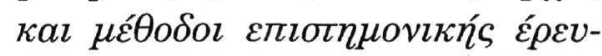

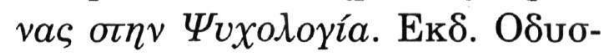

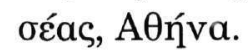

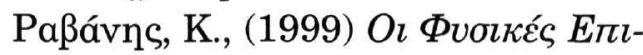

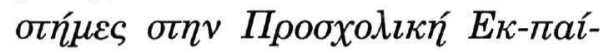

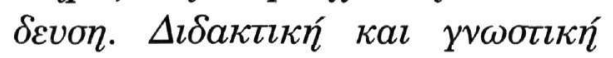

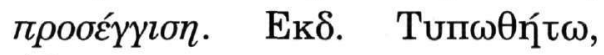
Aөńva. 


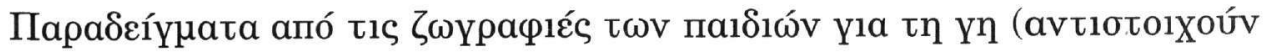
otıৎ катпYopíç tou Пívaka 1).

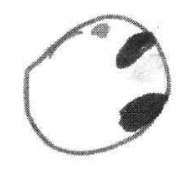

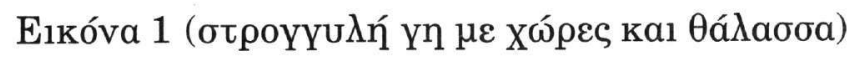

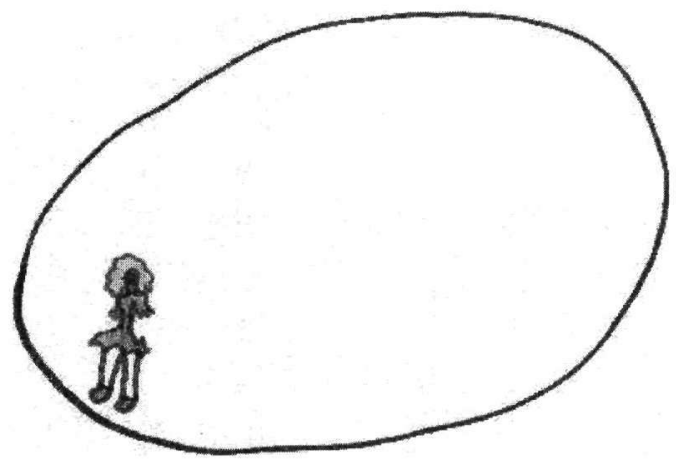

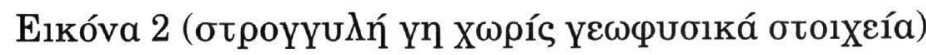

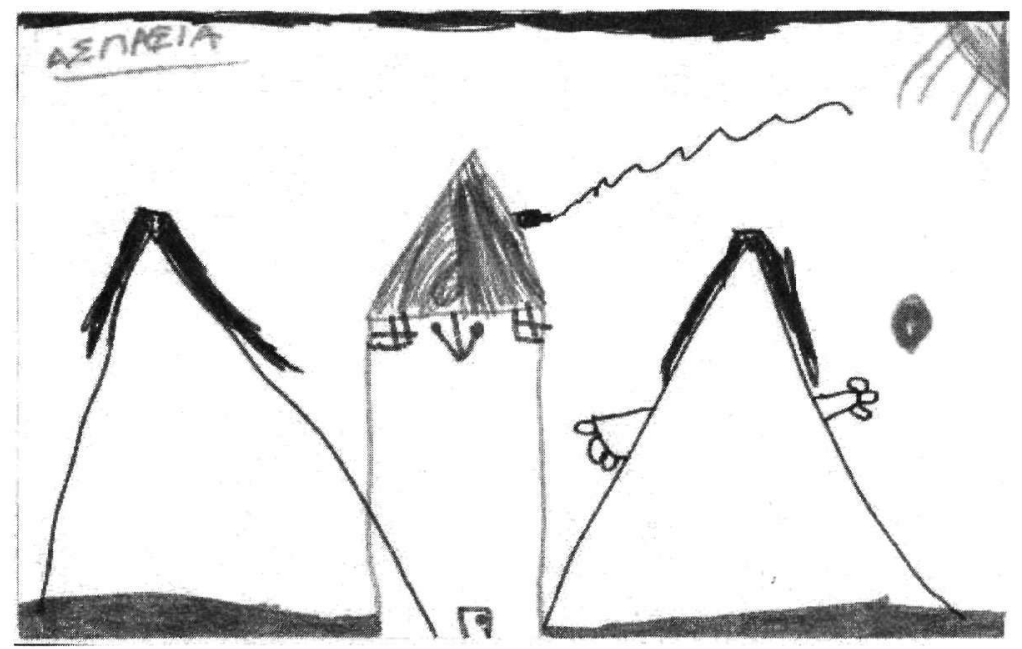

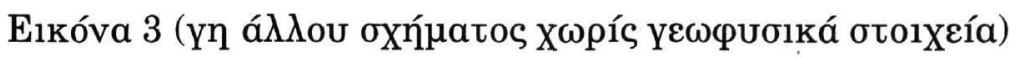




\section{Summary}

There has been intensive research focused on children's alternative conceptions of various natural concepts and phenomena. It is assumed that the acquisition of knowledge occurs as individuals, themselves, construct their own meanings from everyday experiences. These alternative representations are potential constraints on the know-ledge acquisition process, when they are not taken into consideration. The present research is related to the preschoolers' understanding of the earth's surface as a natural space for living. Specifically, earth's shape and geophysical characteristics are examined as well as the macroscopic perspective. The results concerning the earth's shape reveal that although young children are aware that the shape is spherical, they don't relate it with the natural space that they live. In addition, they don't seem to conceptualize the difference between the macroscopic and microscopic perspectives.

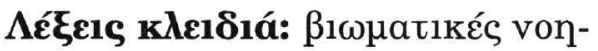

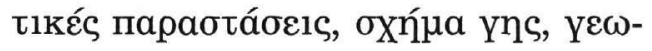

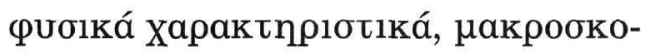

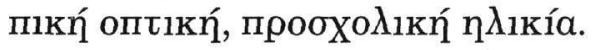

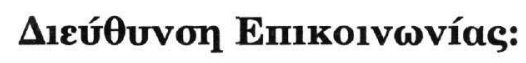

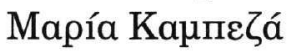

Nikńta 33-37

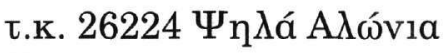

Пát $\rho \alpha$

$\operatorname{T\eta } \Lambda$. (061)346-661

e-mail: kampeza@upatras.gr 